\title{
On the exact and numerical solutions to a new $(2+1)$-dimensional Korteweg-de Vries equation with conformable derivative
}

https://doi.org/10.1515/nleng-2021-0005

Received Aug 14, 2020; accepted Jan 28, 2021.

\begin{abstract}
The aim of this paper is to introduce a novel study of obtaining exact solutions to the $(2+1)$ dimensional conformable KdV equation modeling the amplitude of the shallow-water waves in fluids or electrostatic wave potential in plasmas. The reduction of the governing equation to a simpler ordinary differential equation by wave transformation is the first step of the procedure. By using the improved $\tan (\phi / 2)$ - expansion method (ITEM) and Jacobi elliptic function expansion method, exact solutions including the hyperbolic function solution, rational function solution, soliton solution, traveling wave solution, and periodic wave solution of the considered equation have been obtained. We achieve also a numerical solution corresponding to the initial value problem by conformable variational iteration method (C-VIM) and give comparative results in tables. Moreover, by using Maple, some graphical simulations are done to see the behavior of these solutions with choosing the suitable parameters.
\end{abstract}

Keywords: Solitons, Korteweg-de Vries equation, exact solutions, improved $\tan (\phi / 2)$-expansion method, Jacobi elliptic function expansion method

\section{Introduction}

Nonlinear evolution equations (NLEEs) have been used for many years to express the modern world phenomena we encounter in nonlinear sciences such as mathematical biology, plasma physics, elastic media, finance, fluid mechanics, control theory, chemistry, optics, engineering sciences, etc. The concept of fractional derivative operator

\footnotetext{
*Corresponding Author: Emrullah Yaşar, Department of Mathematics, Faculty of Arts and Sciences, Uludag University, 16059 Bursa, Turkey, E-mail: emrullah.yasar@gmail.com

Yeşim Sağlam Özkan, Nisa Çelik, Department of Mathematics, Faculty of Arts and Sciences, Uludag University, 16059 Bursa, Turkey, E-mail: (ysaglam@uludag.edu.tr, nisacelik@gmail.com)
}

dates back to the work of L'hospital in the 17th century. We need fractional partial differential equations (FPDEs) to be able to and interpret physical models that occur in most applied sciences. Recently, we have been observing these equations, especially in physical models that contain space and time variables. In fact, fractional derivatives better explain the various physical phenomena encountered. It is quite crucial to examine fractional spacetime differential equations which are nonlocal operator instead of integer ordered differential operator which is a local operator. For, as is known, this means that the next state of a system depends not only on its present state but also on all its former states. This is the main advantage of fractional differential equations over integer-order differential equations and this makes the model created more realistic [1]. There are several definitions of fractional derivative, such as Riemann-Liouville, Caputo, CaputoFabrizio, Atangana-Baleanu [2-6].

In 2014, a new derivative called conformable derivative by Khalil [7] was identified and developed by Abdeljavad [8]. To date, there are many studies in the literature on this subject [9-16]. Over the past few years, many methods have been proposed to find exact solutions of equations with fractional derivatives. For example first integral method [17-19], the modified trial equation method [20, 21], auxiliary equation method [22, 23], the modified Kudryashov method [24-26], Jacobi elliptic function expansion method [27] and so on [28-31].

The classical $(1+1)$-dimensional KdV equation

$$
u_{t}+6 u_{x} u+u_{x x x}=0
$$

is a well known equation that is utilized to characterize the waves on shallow water surfaces. In addition, this equation has a wide range of applications in various branches such as bubble liquid mixtures, waves in enharmonic crystals, ion acoustic wave and magneto-hydrodynamic waves in a warm plasma, cold lossless (collisionless) plasma as well as shallow water waves [32-37]. One of the main features of the KdV equation is that the speed of solitary wave is related to the magnitude of the solitary wave, and the other is that the solutions can represent solitary wave solutions noted as solitons which have quantum mechani- 
cal effects which occur in particle physics and quantum field theory [33, 37]. Many researchers have investigated numerous versions of this famous equation with different procedures and techniques [38-49]. A few of the various interesting features of this well-known equation are an infinite number of conservation laws (higher order), bi-Hamilton structures, symmetries and the Lax pair. The $(2+1)$-dimensional $\mathrm{KdV}$ equation

$$
u_{t}+3(u v)_{x}+u_{x x x}=0, u_{x}-v_{y}=0
$$

was derived by Boiti et al. using the idea of the weak Lax pair [50]. For $v=u$ and $y=x$, Eq. (2) reads the $(1+1)$-dimensional $\mathrm{KdV}$ equation. Compared to $(1+1)$ dimensional case, the $(2+1)$-dimensional case explains a more involved nonlinear phenomenon. As highlighted in the lines above that physical systems can be better expressed with fractional order derivatives used for global modeling.

The main objective of this paper is to use ITEM to find the new exact solutions of the time fractional $(2+1)$ dimensional $\mathrm{KdV}$ equation given as folllows

$$
\begin{aligned}
& D_{t}^{\alpha} u-6 u_{x} u+6 u_{y} u-u_{x x x}+u_{y y y}+3 u_{x x y}-3 u_{x y y}=0 \\
& t \geq 0,0<\alpha \leq 1 .
\end{aligned}
$$

where $D_{t}^{\alpha}($.$) is conformable derivative of order \alpha$. When $\alpha=1$, Eq. (3) changes to the $(2+1)$-dimensional KdV equation which has been proved based on the extended Lax pair and has been announced in [46].

The organization of this paper is as follows: In Section 2, some basic definitions of the conformable derivative are recalled. In Sections 3,4 and 5, the key idea of ITEM, Jacobi elliptic function expansion method and conformable variational iteration method is described. In section 6 , the acquisition of the considered physical model is briefly presented. In Sections 7,8 and 9, applications of the methods are given. Finally, some conclusions are presented in the last section.

\section{Conformable derivative}

R. Khalil et al. proposed a derivative that coincides with the classical derivative when $\alpha=1$ and that can rectify the shortcomings of the previous definitions. Here, definition and some properties of the conformable derivative are presented [7, 8].

Definition [7]: Let $f:[0, \infty) \rightarrow \mathbb{R}$. Then the $\alpha$ order conformable derivative of $f$ is defined as

$$
T_{\alpha}(f)(t)=\lim _{\epsilon \rightarrow 0} \frac{f\left(t+\epsilon t^{1-\alpha}\right)-f(t)}{\epsilon}
$$

for all $t>0, \alpha \in(0,1)$.

Theorem 1: Let $T_{\alpha}$ be a conformable derivative operator with order $\alpha$ and $\alpha \in(0,1], f, g$ be $\alpha$-differentiable at point $t>0$. Then $[6,7]$,

(i) $T_{\alpha}(a f+b g)=a T_{\alpha}(f)+b T_{\alpha}(g), \forall a, b \in \mathbb{R}$.

(ii) $T_{\alpha}\left(t^{p}\right)=p t^{p-\alpha}, \forall p \in \mathbb{R}$.

(iii) $T_{\alpha}(f g)=f T_{\alpha}(g)+g T_{\alpha}(f)$.

(iv) $T_{\alpha}\left(\frac{f}{g}\right)=\frac{g T_{\alpha}(f)-f T_{\alpha}(g)}{g^{2}}$.

(v) $T_{\alpha}(\lambda)=0$, for all constant functions $f(t)=\lambda$.

(vi) If $f$ is differentiable, then $T_{\alpha}(f)(t)=t^{1-\alpha} \frac{d f}{d t}(t)$.

\section{Algorithm of the improved $\tan (\phi / 2)$-expansion method for NLEEs}

This method was summarized and improved for achieving the analytic solutions of NLEEs by Manafian et al in 2015 [51]. Assume a nonlinear partial differential equation is given in general form as follows

$$
\mathfrak{N}\left(u, u_{x}, u_{t}, u_{x x}, u_{t t}, \ldots\right)=0 .
$$

After simple algebraic operations, this equation is transformed into an ordinary differential equation (ODE) with $\xi=x-\mu t$ transformation

$$
\mathfrak{Q}\left(u, u^{\prime},-\mu u^{\prime}, u^{\prime \prime}, \mu^{2} u^{\prime \prime}, \ldots\right)=0 .
$$

Then, assume that the searched wave solutions of Eq. (5) have the following representation

$u(\xi)=S(\phi)=\sum_{k=0}^{m} A_{k}[p+\tan (\phi / 2)]^{k}+\sum_{k=1}^{m} B_{k}[p+\tan (\phi / 2)]^{-k}$

where $A_{k}(0 \leq k \leq m)$ and $B_{k}(1 \leq k \leq m)$ are constants to be determined and $p$ is arbitrary constant, such that $A_{m} \neq 0$, $B_{m} \neq 0$ and $\phi=\phi(\xi)$ is the solution of the following first order differential equation:

$$
\phi^{\prime}(\xi)=a \sin (\phi(\xi))+b \cos (\phi(\xi))+c .
$$

If we try to find the solution of the (7), then we obtain special solutions that vary according to the state of the coefficients:

Family 1. When $\Delta=a^{2}+b^{2}-c^{2}<0$ and $b-c \neq 0$, then $\phi(\xi)=2 \tan ^{-1}\left[\frac{a}{b-c}-\frac{\sqrt{-\Delta}}{b-c} \tan \left(\frac{\sqrt{-\Delta}}{2} \bar{\xi}\right)\right]$

Family 2. When $\Delta=a^{2}+b^{2}-c^{2}>0$ and $b-c \neq 0$, then $\phi(\xi)=2 \tan ^{-1}\left[\frac{a}{b-c}+\frac{\sqrt{\Delta}}{b-c} \tanh \left(\frac{\sqrt{\Delta}}{2} \bar{\xi}\right)\right]$

Family 3. When $\Delta=a^{2}+b^{2}-c^{2}>0, b \neq 0$ and $c=0$, then $\phi(\xi)=2 \tan ^{-1}\left[\frac{a}{b}+\frac{\sqrt{b^{2}+a^{2}}}{b} \tanh \left(\frac{\sqrt{b^{2}+a^{2}}}{2} \bar{\xi}\right)\right]$ 
Family 4. When $\Delta=a^{2}+b^{2}-c^{2}<0, c \neq 0$ and $b=0$, then $\phi(\xi)=2 \tan ^{-1}\left[\frac{-a}{c}+\frac{\sqrt{c^{2}-a^{2}}}{c} \tanh \left(\frac{\sqrt{c^{2}-a^{2}}}{2} \bar{\xi}\right)\right]$

Family 5. When $\Delta=a^{2}+b^{2}-c^{2}>0, b-c \neq 0$ and $a=0$, then $\phi(\xi)=2 \tan ^{-1}\left[\sqrt{\frac{b+c}{b-c}} \tanh \left(\frac{\sqrt{b^{2}-c^{2}}}{2} \bar{\xi}\right)\right]$

Family 6. When $a=0$ and $c=0$, then $\phi(\xi)=$ $\tan ^{-1}\left[\frac{e^{2 b \dot{\xi}}-1}{e^{2 b \grave{\xi}}+1}, \frac{e^{2 b \vec{\xi}}}{e^{2 b \dot{\xi}}+1}\right]$

Family 7. When $b=0$ and $c=0$, then $\phi(\xi)=$ $\tan ^{-1}\left[\frac{e^{2 a \bar{\xi}}}{e^{2 a \bar{\xi}}+1}, \frac{e^{2 a \bar{\xi}}-1}{e^{2 a \bar{\xi}}+1}\right]$

Family 8. When $a^{2}+b^{2}=c^{2}$, then $\phi(\xi)=2 \tan ^{-1}\left[\frac{a \bar{\xi}+2}{(b-c) \bar{\xi}}\right]$

Family 9. When $a=b=c=k a$, then $\phi(\xi)=$ $2 \tan ^{-1}\left[e^{k a \bar{\xi}}-1\right]$

Family 10. When $a=c=k a$ and $b=-k a$, then $\phi(\xi)=-2 \tan ^{-1}\left[\frac{e^{k a \xi}}{e^{k a \xi}-1}\right]$

Family 11. When $c=a$, then $\phi(\xi)=-2 \tan ^{-1}\left[\frac{(a+b) e^{b \xi}-1}{(a-b) e^{b \xi}-1}\right]$

Family 12. When $a=c$, then $\phi(\xi)=2 \tan ^{-1}\left[\frac{(c+b) e^{b \xi}+1}{(b-c) e^{b \xi}-1}\right]$

Family 13. When $c=-a$, then $\phi(\xi)=2 \tan ^{-1}\left[\frac{e^{b \xi}+b-a}{e^{b \xi}-b-a}\right]$

Family 14. When $b=-c$, then $\phi(\xi)=2 \tan ^{-1}\left[\frac{a e^{a \bar{\xi}}}{1-d e^{a \xi}}\right]$

Family 15. When $b=0$ and $a=c$, then $\phi(\xi)=$ $-2 \tan ^{-1}\left[\frac{c \bar{\xi}+2}{c \bar{\xi}}\right]$

Family 16. When $a=0$ and $b=c$, then $\phi(\xi)=2 \tan ^{-1}[c \bar{\xi}]$

Family 17. When $a=0$ and $b=-c$, then $\phi(\xi)=$ $-2 \tan ^{-1}\left[\frac{1}{c \bar{\xi}}\right]$

Family 18. When $a=0$ and $b=0$, then $\phi(\xi)=c \xi+C$

Family 19. When $b=c$, then $\phi(\xi)=2 \tan ^{-1}\left[\frac{e^{a \xi_{\xi}}-c}{a}\right]$, where $\bar{\xi}=\xi+C, p, A_{0}, A_{k}, B_{k}(k=1,2, \ldots, m), a, b$ and $c$ are constants to be determined later.

As usual, for determining $m$, the highest order derivative should be balanced with the highest order nonlinear terms in Eq. (5). In the case of $m=q / p$ (where $m=q / p$ be a fraction in the lowest term), we need to do a conversion on the unknown function $u$ as follows:

$$
u(\xi)=(v(\xi))^{q / p} .
$$

Then substitute Eq. (8) into Eq. (5). By using of new Eq. (5), the value of $m$ can be determined. If $m$ be a negative integer, similar process can be followed with the transformation

$$
u(\xi)=(v(\xi))^{m} .
$$

Following these operations, according to the $m$ value obtained above, let substitute (6) into Eq. (5). Therefore we obtain a set of algebraic equations that contains $\tan (\phi / 2)^{k}, \cot (\phi / 2)^{k},(k=0,1,2, \ldots)$. Then setting each coefficients of $\tan (\phi / 2)^{k}, \cot (\phi / 2)^{k}$ to zero, we can get a set of over-determined equations for $A_{0}, A_{k}, B_{k}(k=$
$1,2, \ldots, m), a, b, c$ and $p$. Since obtained algebraic equations system will be difficult to solve manually, symbolic computation as Maple can be used at this stage. Finally, $A_{0}, A_{1}, B_{1}, \ldots, A_{m}, B_{m}, \mu, p$ are replaced in the Eq. (6).

\section{Algorithm of the Jacobi elliptic function expansion method}

In this section, we recall the Jacobi elliptic function expansion method [52]. Consider a nonlinear partial differential equation is

$$
\mathfrak{N}\left(u, u_{x}, u_{t}, u_{x x}, u_{t t}, \ldots\right)=0 .
$$

Applying the transformation $\xi=x-\mu t$, (9) is transformed into an ODE

$$
\mathfrak{Q}\left(u, u^{\prime},-\mu u^{\prime}, u^{\prime \prime}, \mu^{2} u^{\prime \prime}, \ldots\right)=0,
$$

where $u=u(\xi), u^{\prime}=\frac{d u}{d \xi}, \ldots$. In order to construct more general periodic and solitary wave solution of Eq. (3) by employing the Jacobi elliptic function expansion method, it is assumed that $u(\xi)$ can be formulated as a finite series of Jacobi elliptic sine and cosine functions. The ansatz are given below

$$
u(\xi)=\sum_{j=0}^{n} a_{j} s n^{j}(\xi)
$$

and

$$
u(\xi)=\sum_{j=0}^{n} b_{j} c n^{j}(\xi),
$$

where $n, a_{j}$ and $b_{j}(j=0,1,2,3, \ldots)$ are constants. $s n(\xi)=$ $s n(\xi \mid m)$ and $c n(\xi)=c n(\xi \mid m)$ where $m(0<m<1)$ is called a modulus of the elliptic function, are double periodic and satisfy the following properties:

$$
\begin{aligned}
s n^{2}(\xi)+c n^{2}(\xi) & =1, d n^{2}(\xi)+m^{2} s n^{2}(\xi)=1, \\
\frac{d}{d \xi} s n(\xi) & =c n(\xi) d n(\xi), \frac{d}{d \xi} c n(\xi)=-s n(\xi) d n(\xi), \\
\frac{d}{d \xi} d n(\xi) & =-m^{2} c n(\xi) \operatorname{sn}(\xi) .
\end{aligned}
$$

The value of $n$ is determined againby balancing the nonlinear term and the highest derivative. Therefore, the highest degree of $\frac{d^{p} u}{d \xi^{p}}$ is taken as

$$
O\left(\frac{d^{p} u}{d \xi^{p}}\right)=n+p, \quad p=1,2,3, \ldots
$$

and the nonlinear term as

$$
O\left(u^{q} \frac{d^{p} u}{d \xi^{p}}\right)=(q+1) n+p, \quad q=0,1,2,3, \ldots .
$$


Then substituting the ansatz (11) and (12) into Eq. (10) and equating the coefficients of all powers of elliptic functions to zero, we get a system of algebraic equations for $a_{j}$ and $b_{j}(j=0,1,2,3, \ldots)$.

\section{Succint overview of the conformable variational iteration method}

In this section, we will present how the conformable variational iteration method (C-VIM) works for conformable nonlinear evolution type equations $[53,54]$. Let us assume that the following conformable nonlinear evolution equations in operator form

${ }_{t} T_{\alpha} u(x, t)+L(u(x, t))+N(u(x, t))=g(x, t), n<\alpha \leq n+1$

where $L$ is a linear operator, $N$ is a non-linear operator, $g$ is an non-homogeneous term and ${ }_{t} T_{\alpha}$ is conformable derivative of order $\alpha$. To solve differential equation (15) via C-VIM write the differential equation (15) in the form by Theorem 1 (property (vi)),

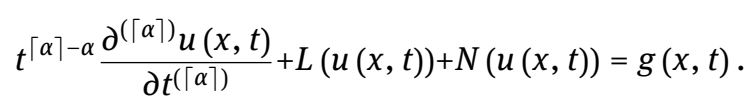

As in classical variational iteration method, the trial functional for (16) can be constructed as

$$
\begin{aligned}
& u_{n+1}(x, t)=u_{n}(x, t)+\int_{0}^{t} \lambda(\zeta)\left[\zeta^{\lceil\alpha\rceil-\alpha} \frac{\partial^{(\lceil\alpha\rceil)} u_{n}(x, \zeta)}{\partial \zeta(\lceil\alpha\rceil)}\right. \\
& \left.+L\left(u_{n}(x, \zeta)\right)+N\left(\tilde{u}_{n}(x, \zeta)\right)-g(x, \zeta)\right] d \zeta
\end{aligned}
$$

where $\lambda$ is a general Lagrangian multiplier and it can be optimally determined by the aid of variational theory [5557]. Here $\tilde{u}_{n}$ is a restricted variation [55-57] where $\delta \tilde{u}_{n}=$ 0 . As the first step of this approach, $\lambda$ multiplier should bederived by the help of variational theory and integration by parts. Using the determined Lagrangian multiplier and any selected function $u_{0}$, the $u_{n+1}$ iteration, which is the successive approximations of $u(x)$ for $n \geq 0$, will be obtained readily. Hence, we get the solution as

$$
u(x, t)=\lim _{n \rightarrow \infty} u_{n}(x, t) .
$$

\section{Governing equation}

Recently in 2019, a new (2+1)-dimensional KdV equation has been proved based on the extended Lax pair [46]. To derive of the $(2+1)$-dimensional $\mathrm{KdV}$ equation, $(2+1)$ dimensional zero curvature equation $[42,58,59]$ considered

$$
X_{t}-X_{X}+T_{X}-T_{y}+X T-T X=0,
$$

where

$$
X=\left(\begin{array}{cc}
-i \zeta & q \\
r & i \zeta
\end{array}\right), T=\left(\begin{array}{cc}
A & B \\
C & D
\end{array}\right),
$$

with the compatibility conditions and $\zeta_{t}=0$. Plugging (18) into Eq. (17), a system of algebraic equations is obtained. Based on the work done by Ablowitz [42], $A, B, C, D$ is assumed to have a series expansion in $K=\sum_{j=0}^{n} k_{i}(x, y, t) \zeta^{n-j}$, where $K=A, B, C, D$ and $k=$ $a, b, c, d$. Relations between coefficients is found by using algebraic equation system and series expansions of functions [46]. If $n=2$, the new $(2+1)$-dimensional Schrödinger equation [59] is obtained

$$
i\left(q_{x}-q_{t}\right)+q_{x x}+q_{y y}-2 q_{x y}+2|q|^{2} q=0 .
$$

If $n=3$, with the appropriate selection of coefficients [46], it immediately generates the following new $(2+1)$ dimensional KdV equation

$$
q_{t}-q_{x}-6 q q_{x}+6 q q_{y}-q_{x x x}+q_{y y y}+3 q_{x x y}-3 q_{x y y}=0 .
$$

If $q=u$, by the help of the Galilean transformation $X=$ $x-t, T=t, Y=y$, one can get

$$
u_{t}-6 u u_{x}+6 u u_{y}-u_{x x x}+u_{y y y}+3 u_{x x y}-3 u_{x y y}=0 .
$$

If $y=x,(20)$ is reduced to $(1+1)$-dimensional KdV equation (1). In the next sections, the $(2+1)$-dimensional $\mathrm{KdV}$ equation with conformable derivative (3) obtained via Eq.

(20) will be discussed .

\section{Application of ITEM to conformable $(2+1)$ dimensional $\mathrm{KdV}$ equation}

In this section, we apply the ITEM to Eq. (3) to obtain the traveling wave solutions. In this context, let us consider $u(x, t)=u(\xi), \xi=k x+r y-\frac{w}{\alpha} t^{\alpha}$ and therefore Eq. (3) becomes

$$
-w u^{\prime}-(6 k-6 r) u^{\prime} u-\left(k^{3}-r^{3}-3 r k^{2}+3 r^{2} k\right) u^{\prime \prime \prime}=0
$$

where " ' " shows the derivative according to $\xi$. By integrating (21) once with respect to $\xi$, we obtain

$$
-w u-(3 k-3 r) u^{2}-\left(k^{3}-r^{3}-3 r k^{2}+3 r^{2} k\right) u^{\prime \prime}=0 .
$$


With balancing prodecure, where $u^{\prime \prime}$ derivative is balanced by $u^{2}, m+2=2 m$, then $m=2$ is obtained. Therefore, by considering $p=0$ in (6), we get the following finite series expansion for unknown function of $u(\xi)$

$$
u(\xi)=A_{0}+A_{1} \tan \left(\frac{\phi(\xi)}{2}\right)+A_{2}\left(\tan \left(\frac{\phi(\xi)}{2}\right)\right)^{2}+B_{1}\left(\cot \left(\frac{\phi(\xi)}{2}\right)\right)+B_{2}\left(\cot \left(\frac{\phi(\xi)}{2}\right)\right)^{2} .
$$

We substitute the expression of $u$ in (23) into (22) and collect all terms with the same order of $\tan (\phi(\xi) / 2), \cot (\phi(\xi) / 2)$ together. Then by equating the coefficient of each polynomial to zero, we obtain a set of algebraic equations

$$
\begin{gathered}
3 B_{2} b^{2} k^{3}-9 B_{2} b^{2} k^{2} r+9 B_{2} b^{2} k r^{2}-3 B_{2} b^{2} r^{3}+6 B_{2} b c k^{3}-18 B_{2} b c k^{2} r+18 B_{2} b c k r^{2} \\
-6 B_{2} b c r^{3}+3 B_{2} c^{2} k^{3}-9 B_{2} c^{2} k^{2} r+9 B_{2} c^{2} k r^{2}-3 B_{2} c^{2} r^{3}+6 B_{2}{ }^{2} k-6 B_{2}^{2} r=0, \\
2 B_{1} b^{2} k^{3}-6 B_{1} b^{2} k^{2} r+6 B_{1} b^{2} k r^{2}-2 B_{1} b^{2} r^{3}+4 B_{1} b c k^{3}-12 B_{1} b c k^{2} r-24 B_{1} B_{2} r \\
+12 B_{1} b c k r^{2}-4 B_{1} b c r^{3}+2 B_{1} c^{2} k^{3}-6 B_{1} c^{2} k^{2} r+6 B_{1} c^{2} k r^{2}-2 B_{1} c^{2} r^{3}+24 B_{1} B_{2} k \\
+20 B_{2} a b k^{3}-60 B_{2} a b k^{2} r+60 B_{2} a b k r^{2}-20 B_{2} a b r^{3}+20 B_{2} a c k^{3}-20 B_{2} a c r^{3} \\
-60 B_{2} a c k^{2} r+60 B_{2} a c k r^{2}=0,
\end{gathered}
$$

Solving the above algebraic equations (24) by help of Maple, we have numerous sets of coefficients for the solutions of (22). We only choose some of them as follows:

\section{SET 1}

We have yielded the arbitrary constants as

$$
\left\{\begin{array}{c}
A_{0}=\frac{1}{6} r^{2} b^{2}-\frac{1}{6} k^{2} c^{2}+\frac{1}{6} k^{2} b^{2}-\frac{1}{6} r^{2} c^{2}-\frac{1}{3} k r b^{2}+\frac{1}{3} k r c^{2}, A_{1}=0 \\
A_{2}=-\frac{1}{2}(-b r+k b+r c-k c)^{2}, B_{1}=0, B_{2}=0, a=0, b=b, c=c, k=k, r=r \\
w=k^{3} b^{2}-3 r k^{2} b^{2}+3 r^{2} k b^{2}-r^{3} b^{2}-k^{3} c^{2}+3 r k^{2} c^{2}-3 r^{2} k c^{2}+r^{3} c^{2}
\end{array}\right\}
$$

By using Family 1, (23) becomes

$u_{1}(x, y, t)=\frac{(k-r)^{2}(b-c)(b+c)}{6}-\frac{(-b r+k b+r c-k c)^{2}\left(-b^{2}+c^{2}\right)\left(\tan \left(\frac{\sqrt{-b^{2}+c^{2}}}{2}\left(k x+r y-\frac{(k-r)^{3}(b-c)(b+c) t^{\alpha}}{\alpha}+C\right)\right)\right)^{2}}{2(b-c)^{2}}$.

By using Family 2, (23) reads

$$
\begin{aligned}
u_{2}(x, y, t)= & \frac{(k-r)^{2}(b-c)(b+c)}{6} \\
& -\frac{(k-r)^{2}(b-c)(b+c)}{2}\left(\tanh \left(\frac{\sqrt{b^{2}-c^{2}}}{2}\left(k x+r y-\frac{(k-r)^{3}(b-c)(b+c) t^{\alpha}}{\alpha}+C\right)\right)\right)^{2} .
\end{aligned}
$$

By using Family 5 , one constructs for (23)

$$
\begin{aligned}
u_{3}(x, y, t)= & \frac{(k-r)^{2}\left(b^{2}-c^{2}\right)}{6} \\
& -\frac{(-b r+k b+r c-k c)^{2}}{2} \frac{(b+c)}{b-c}\left(\tanh \left(\frac{\sqrt{b^{2}-c^{2}}}{2}\left(k x+r y-\frac{(k-r)^{3}(b-c)(b+c) t^{\alpha}}{\alpha}+C\right)\right)\right)^{2} .
\end{aligned}
$$

By using Family 8, (23) can be written as

$$
u_{4}(x, y, t)=-\frac{-24 k r+12 r^{2}+12 k^{2}}{6(k x+r y+C)^{2}} .
$$

By using Family 11, we can write

$$
u_{5}(x, y, t)=\frac{(k-r)^{2} b^{2}}{6}-\frac{\frac{(-b r+k b)^{2}}{2}\left(b e^{b\left(k x+r y-\frac{(k-r)^{3} b^{2} t^{\alpha}}{\alpha}+C\right)}-1\right)^{2}}{\left(-b e^{b\left(k x+r y-\frac{(k-r)^{3} b^{2} t^{\alpha}}{\alpha}+C\right)}-1\right)^{2}} .
$$


By using Family 12, (23) becomes

$$
u_{6}(x, y, t)=\frac{(k-r)^{2} b^{2}}{6}-\frac{(-b r+k b)^{2}\left(b e^{b\left(k x+r y-\frac{(k-r)^{3} b^{2} t^{\alpha}}{\alpha}+C\right)}+1\right)^{2}}{2\left(b e^{b\left(k x+r y-\frac{(k-r)^{3} b^{2} t^{\alpha}}{\alpha}+C\right)}-1\right)^{2}} .
$$

By using Family 13, (23) reads

$$
u_{7}(x, y, t)=\frac{(k-r)^{2} b^{2}}{6}-\frac{(-b r+k b)^{2}\left(e^{b\left(k x+r y-\frac{(k-r)^{3} b^{2} t^{\alpha}}{\alpha}+C\right)}+b\right)^{2}}{2\left(e^{b\left(k x+r y-\frac{(k-r) b^{3} b^{2} t^{\alpha}}{\alpha}+C\right)}-b\right)^{2}} .
$$

By using Family 17, one constructs for (23)

$$
u_{8}(x, y, t)=-\frac{(2 r-2 k)^{2}}{2(k x+r y+C)^{2}}
$$

By using Family 18, we get

$$
u_{9}(x, y, t)=-\frac{(k-r)^{2} c^{2}}{6}-\frac{(r-k)^{2} c^{2}}{2}\left(\tan \left(\frac{c}{2}\left(k x+r y+\frac{(k-r)^{3} c^{2} t^{\alpha}}{\alpha}\right)+\frac{C}{2}\right)\right)^{2} .
$$

\section{SET 2}

We have yielded the arbitrary constants as

$$
\left\{\begin{array}{c}
A_{0}=\frac{(-r+k)^{2}\left(b^{2}-c^{2}\right)}{2}, A_{1}=A_{1}, A_{2}=-\frac{(-r+k)^{2}(-c+b)^{2}}{2}, B_{1}=0, B_{2}=0 \\
a=\frac{A_{1}}{(-r+k)^{2}(-c+b)}, b=b, c=c, k=k, r=r, w=\frac{-(-r+k)^{4}(b+c)(-c+b)^{3}+A_{1}^{2}}{(-r+k)(-c+b)^{2}}
\end{array}\right\}
$$

By using Family 1, (23) can be written as

$$
\begin{aligned}
u_{10}(x, y, t)= & \frac{(-r+k)^{2}(-c+b)(b+c)}{2}+A_{1}\left(\frac{A_{1}}{(-r+k)^{2}(-c+b)^{2}}-\sqrt{c^{2}-b^{2}-\frac{A_{1}^{2}}{(-c+b)^{2}(-r+k)^{4}(-c+b)} \frac{\tan (F)}{(-c+b)}}\right) \\
& -\frac{(-r+k)^{2}(-c+b)^{2}}{2}\left(\frac{A_{1}}{(-r+k)^{2}(-c+b)^{2}}-\sqrt{c^{2}-b^{2}-\frac{A_{1}^{2}}{(-c+b)^{2}(-r+k)^{4}} \frac{\tan (F)}{(-c+b)}}\right)^{2},
\end{aligned}
$$

where

$$
F=\frac{\sqrt{c^{2}-b^{2}-\frac{A_{1}^{2}}{(-c+b)^{2}(-r+k)^{4}}}\left(k x+r y+\frac{\left((-r+k)^{4}(b+c)(-c+b)^{3}+A_{1}^{2}\right) t^{\alpha}}{(-r+k)(-c+b)^{2} \alpha}+C\right)}{2} .
$$

By using Family 2, (23) becomes

$$
\begin{aligned}
u_{11}(x, y, t)= & \frac{(-r+k)^{2}(-c+b)(b+c)}{2}+A_{1}\left(\frac{A_{1}}{(-r+k)^{2}(-c+b)^{2}}+\sqrt{-c^{2}+b^{2}+\frac{A_{1}^{2}}{(-c+b)^{2}(-r+k)^{4}}} \frac{\tan (F)}{(-c+b)}\right) \\
& -\frac{(-r+k)^{2}(-c+b)^{2}}{2}\left(\frac{A_{1}}{(-r+k)^{2}(-c+b)^{2}}+\sqrt{-c^{2}+b^{2}+\frac{A_{1}^{2}}{(-c+b)^{2}(-r+k)^{4}} \frac{\tan (F)}{(-c+b)}}\right)^{2},
\end{aligned}
$$

where

$$
F=\frac{\sqrt{-c^{2}+b^{2}+\frac{A_{1}^{2}}{(-c+b)^{2}(-r+k)^{4}}}}{2}\left(k x+r y+\frac{\left((-r+k)^{4}(b+c)(-c+b)^{3}+A_{1}^{2}\right) t^{\alpha}}{(-r+k)(-c+b)^{2} \alpha}+C\right) .
$$

By using Family 3, we obtain

$$
u_{12}(x, y, t)=\frac{(-r+k)^{2} b^{2}}{2}+A_{1}\left(\frac{A_{1}}{(-r+k)^{2} b^{2}}+\frac{1}{b} \sqrt{b^{2}+\frac{A_{1}^{2}}{b^{2}(-r+k)^{4}}} \tanh (F)\right)
$$




$$
-1 / 2(-r+k)^{2} b^{2}\left(\frac{A_{1}}{(-r+k)^{2} b^{2}}+\frac{1}{b} \sqrt{b^{2}+\frac{A_{1}^{2}}{b^{2}(-r+k)^{4}}} \tanh (F)\right)^{2}
$$

where

$$
F=\frac{\sqrt{b^{2}+\frac{A_{1}^{2}}{b^{2}(-r+k)^{4}}}}{2}\left(k x+r y+\frac{\left((-r+k)^{4} b^{4}+A_{1}^{2}\right) t^{\alpha}}{(-r+k) b^{2} \alpha}+C\right) .
$$

By using Family 4, one constructs for (23)

$$
\begin{aligned}
u_{13}(x, y, t)= & -\frac{(-r+k)^{2} c^{2}}{2}+A_{1}\left(\frac{A_{1}}{(-r+k)^{2} c^{2}}+\frac{1}{c} \sqrt{c^{2}-\frac{A_{1}^{2}}{c^{2}(-r+k)^{4}}} \tan (F)\right) \\
& -\frac{(-r+k)^{2} c^{2}}{2}\left(\frac{A_{1}}{(-r+k)^{2} c^{2}}+\frac{1}{c} \sqrt{c^{2}-\frac{A_{1}^{2}}{c^{2}(-r+k)^{4}}} \tan (F)\right)^{2},
\end{aligned}
$$

where

$$
F=\frac{\sqrt{c^{2}-\frac{A_{1}^{2}}{c^{2}(-r+k)^{4}}}}{2}\left(k x+r y+\frac{\left(-c^{4}(-r+k)^{4}+A_{1}^{2}\right) t^{\alpha}}{(-r+k) c^{2} \alpha}+C\right) .
$$

By using Family 5, (23) can be written as

$$
u_{14}(x, y, t)=\frac{(-r+k)^{2}\left(b^{2}-c^{2}\right)}{2}\left(1-\left(\tanh \left(\frac{\sqrt{b^{2}-c^{2}}}{2}\left(k x+r y+\frac{(-r+k)^{3}(b+c)(-c+b) t^{\alpha}}{\alpha}+C\right)\right)\right)^{2}\right) .
$$

By using Family 11, we can write

$$
u_{15}(x, y, t)=-\frac{2(-r+k)^{2}(a-b) e^{b\left(k x+r y+\frac{b^{2}(-r+k)^{3} t^{\alpha}}{\alpha}+C\right)} b^{2}}{\left((a-b) e^{b\left(k x+r y+\frac{b(-r+k)^{3} t^{\alpha}}{\alpha}+C\right)}-1\right)^{2}} .
$$

By using Family 12, (23) reads

$$
u_{16}(x, y, t)=-\frac{2(-r+k)^{2}(-c+b) e^{b\left(k x+r y+\frac{b^{2}(-r+k)^{3} t^{\alpha}}{\alpha}+c\right)} b^{2}}{\left((b-c) e^{b\left(k x+r y+\frac{b^{2}(-r+k)^{3} t^{\alpha}}{\alpha}+C\right)}-1\right)^{2}} .
$$

By using Family 13, one constructs for (23)

$$
u_{17}(x, y, t)=-\frac{2(-r+k)^{2}(a+b) b^{2} e^{b\left(k x+r y+\frac{b^{2}(-r+k)^{3} t^{\alpha}}{\alpha}+C\right)}}{\left(e^{b\left(k x+r y+\frac{b^{2}(-r+k)^{3} t^{\alpha}}{\alpha}+C\right)}-b-a\right)^{2}} .
$$

By using Family 14, we get

$$
u_{18}(x, y, t)=-\frac{A_{1}^{2} e^{F}}{2 c(-r+k)^{2}\left(-1+c e^{F}\right)^{2}},
$$

where

$$
F=-\frac{A_{1}\left(4(-r+k) c^{2} \alpha(k x+r y+C)+A_{1}^{2} t^{\alpha}\right)}{c^{3}(-r+k)^{3} \alpha} .
$$

By using Family 15, we can write

$$
u_{19}(x, y, t)=-2 \frac{(-r+k)^{2}}{(k x+r y+C)^{2}} .
$$


By using Family 17, (23) becomes

$$
u_{20}(x, y, t)=-2 \frac{(-r+k)^{2}}{(k x+r y+C)^{2}}
$$

By using Family 18, (23) reads

$$
u_{21}=-\frac{(-r+k)^{2} c^{2}}{2}-\frac{(-r+k)^{2} c^{2}}{2}\left(\tan \left(\frac{c}{2}\left(k x+r y-\frac{(-r+k)^{3} c^{2} t^{\alpha}}{\alpha}\right)+\frac{C}{2}\right)\right)^{2} .
$$

\section{SET 3}

We have yielded the arbitrary constants as

$$
\left\{\begin{array}{c}
A_{0}=-\frac{(k-r)^{2}\left(c^{2}-b^{2}+2 a^{2}\right)}{6}, A_{1}=0, A_{2}=0, B_{1}=-a(k-r)^{2}(b+c), \\
B_{2}=-\frac{(k-r)^{2}(b+c)^{2}}{2}, a=a, b=b, c=c, k=k, r=r, w=(k-r)^{3}\left(b^{2}-c^{2}+a^{2}\right)
\end{array}\right\}
$$

By using Family 1, (23) can be written as

$$
u_{22}(x, t)=\frac{(k-r)^{2}\left(\left(b^{2}-c^{2}+a^{2}\right)\left(\left(c^{2}-b^{2}+2 a^{2}\right)(\tan (F))^{2}+4 a \sqrt{-b^{2}+c^{2}-a^{2}} \tan (F)-\left(3 b^{2}+2 a^{2}-3 c^{2}\right)\right)\right)}{6\left(-a+\sqrt{-b^{2}+c^{2}-a^{2}} \tan (F)\right)^{2}},
$$

where

$$
F=\frac{\sqrt{-b^{2}+c^{2}-a^{2}}}{2}\left(k x+r y-\frac{(k-r)^{3}\left(b^{2}-c^{2}+a^{2}\right) t^{\alpha}}{\alpha}+C\right) .
$$

By using Family 2, (23) becomes

$$
u_{23}(x, y, t)=-\frac{(k-r)^{2}\left(b^{2}-c^{2}+a^{2}\right)\left(\left(c^{2}-b^{2}+2 a^{2}\right)(\tanh (F))^{2}+4 a \tanh (F)+3 b^{2}+2 a^{2}-3 c^{2}\right)}{6\left(a+\sqrt{b^{2}-c^{2}+a^{2}} \tanh (F)\right)^{2}},
$$

where

$$
F=\frac{\sqrt{b^{2}-c^{2}+a^{2}}}{2}\left(k x+r y-\frac{(k-r)^{3}\left(b^{2}-c^{2}+a^{2}\right) t^{\alpha}}{\alpha}+C\right) .
$$

By using Family 8, (23) reads

$$
u_{24}(x, y, t)=-\frac{2(k-r)^{2} a^{2}}{(a k x+a r y+a C+2)^{2}} .
$$

By using Family 11, one constructs for (23)

$$
u_{25}(x, y, t)=-\frac{(k-r)^{2}}{3} \frac{\left(\left(4 b^{2} a+4 b^{3}\right) e^{b\left(k x+r y-\frac{(k-r)^{3} b^{2} t^{\alpha}}{\alpha}+C\right)}+\left(b^{4}+a^{2} b^{2}+2 b^{3} a\right) e^{2 b\left(k x+r y-\frac{(k-r)^{3} b^{2} t^{\alpha}}{\alpha}+c\right)}+b^{2}\right)}{\left((a+b) e^{b\left(k x+r y-\frac{(k-r)^{3} b^{2} t^{\alpha}}{\alpha}+c\right)}-1\right)^{2}} .
$$

By using Family 12, (23) can be written as

$$
u_{26}(x, y, t)=-\frac{(k-r)^{2}}{3} \frac{\left(\left(-4 b^{2} c-4 b^{3}\right) e^{b\left(k x+r y-\frac{(k-r)^{3} b^{2} t^{\alpha}}{\alpha}+c\right)}+\left(c^{2} b^{2}+2 b^{3} c+b^{4}\right) e^{2 b\left(k x+r y-\frac{(k-r)^{3} b^{2} t^{\alpha}}{\alpha}+c\right)}+b^{2}\right)}{\left(1+(b+c) e^{b\left(k x+r y-\frac{(k-r)^{3} b^{2} t^{\alpha}}{\alpha}+c\right)}\right)^{2}} .
$$

By using Family 13, (23) can be written as

$$
u_{27}(x, y, t)=\frac{(k-r)^{2}}{3} \frac{\left(\left(-4 a b^{2}+4 b^{3}\right) e^{b\left(k x+r y-\frac{(k-r)^{3} b^{2} t^{\alpha}}{\alpha}+C\right)}-a^{2} b^{2}+2 b^{3} a-b^{4}-b^{2} e^{2 b\left(k x+r y-\frac{(k-r)^{3} b^{2} t^{\alpha}}{\alpha}+C\right)}\right)}{\left(e^{b\left(k x+r y-\frac{(k-r) b^{3} t^{2} t^{\alpha}}{\alpha}+C\right)}+b-a\right)^{2}} .
$$


By using Family 15, (23) reads

$$
u_{28}(x, y, t)=-2 \frac{(k-r)^{2} c^{2}}{(c(k x+r y)+c C+2)^{2}}
$$

By using Family 16, (23) becomes

$$
u_{29}(x, y, t)=-2 \frac{(k-r)^{2}}{(k x+r y+C)^{2}}
$$

By using Family 18, one constructs for (23)

$$
u_{30}(x, y, t)=-\frac{(k-r)^{2}}{6}\left(c^{2}+3\left(\cot \left(\frac{c}{2}\left(k x+r y+\frac{(k-r)^{3} c^{2} t^{\alpha}}{\alpha}\right)+\frac{C}{2}\right)\right)^{2} c^{2}\right) .
$$

By using Family 19, (23) can be written as

$$
u_{31}(x, y, t)=-\frac{(k-r)^{2}}{6} \frac{\left(2 a^{2} e^{2 a\left(k x+r y-\frac{(k-r)^{3} a^{2} t^{\alpha}}{\alpha}+c\right)}+8 a^{2} e^{a\left(k x+r y-\frac{(k-r)^{3} a^{2} t^{\alpha}}{\alpha}+c\right)} c+2 a^{2} c^{2}\right)}{\left(e^{a\left(k x+r y-\frac{(k-r)^{3} a^{2} t^{\alpha}}{\alpha}+c\right)}-c\right)^{2}} .
$$

\section{SET 4:}

We have yielded the arbitrary constants as

$$
\left\{\begin{array}{c}
A_{0}=\frac{(k-r)^{2}\left(b^{2}-c^{2}\right)}{2}, A_{1}=0, A_{2}=0, B_{1}=-a(k-r)^{2}(b+c), \\
B_{2}=-\frac{(k-r)^{2}(b+c)^{2}}{2}, a=a, b=b, c=c, k=k, r=r, w=-(k-r)^{3}\left(b^{2}-c^{2}+a^{2}\right)
\end{array}\right\}
$$

By using Family 1, (23) becomes

$$
\begin{aligned}
u_{32}(x, y, t)= & \frac{(k-r)^{2}\left(b^{2}-c^{2}\right)}{2}-\frac{a(k-r)^{2}\left(b^{2}-c^{2}\right)}{a-\sqrt{-b^{2}+c^{2}-a^{2}} \tan \left(\frac{\sqrt{-b^{2}+c^{2}-a^{2}}}{2}\left(k x+r y+\frac{(k-r)^{3}\left(b^{2}-c^{2}+a^{2}\right) t^{\alpha}}{\alpha}+C\right)\right)} \\
& -\frac{(k-r)^{2}\left(b^{2}-c^{2}\right)^{2}}{2\left(a-\sqrt{-b^{2}+c^{2}-a^{2}} \tan \left(\frac{\sqrt{-b^{2}+c^{2}-a^{2}}}{2}\left(k x+r y+\frac{(k-r)^{3}\left(b^{2}-c^{2}+a^{2}\right) t^{\alpha}}{\alpha}+C\right)\right)\right)^{2}} .
\end{aligned}
$$

By using Family 2, (23) reads

$$
\begin{aligned}
u_{33}(x, y, t)= & \frac{(k-r)^{2}\left(b^{2}-c^{2}\right)}{2}-\frac{a(k-r)^{2}\left(b^{2}-c^{2}\right)}{a+\sqrt{b^{2}-c^{2}+a^{2}} \tanh \left(\frac{\sqrt{b^{2}-c^{2}+a^{2}}}{2}\left(k x+r y+\frac{(k-r)^{3}\left(b^{2}-c^{2}+a^{2}\right) t^{\alpha}}{\alpha}+C\right)\right)} \\
& -\frac{(k-r)^{2}\left(b^{2}-c^{2}\right)^{2}}{2\left(a+\sqrt{b^{2}-c^{2}+a^{2}} \tanh \left(\frac{\sqrt{-b^{2}+c^{2}-a^{2}}}{2}\left(k x+r y+\frac{(k-r)^{3}\left(b^{2}-c^{2}+a^{2}\right) t^{\alpha}}{\alpha}+C\right)\right)\right)^{2}} .
\end{aligned}
$$

By using Family 5, (23) can be written as

$$
u_{34}(x, y, t)=\frac{(k-r)^{2}\left(b^{2}-c^{2}\right)}{2}-\frac{(k-r)^{2}\left(b^{2}-c^{2}\right)}{2}\left(\tanh \left(\frac{\sqrt{b^{2}-c^{2}}}{2}\left(k x+r y+\frac{(k-r)^{3}\left(b^{2}-c^{2}\right) t^{\alpha}}{\alpha}+C\right)\right)\right)^{-2} .
$$

By using Family 8, one constructs for (23)

$$
u_{35}(x, y, t)=-2 \frac{(k-r)^{2} a^{2}}{(a k x+a r y+a C+2)^{2}} .
$$

By using Family 11, (23) reads

$$
u_{36}(x, y, t)=\frac{(k-r)^{2}\left(b^{2}-a^{2}\right)}{2}+a(k-r)^{2}(a+b)\left(\frac{(a-b) e^{b\left(k x+r y+\frac{(k-r)^{3} b^{2} t^{\alpha}}{\alpha}+c\right)}-1}{(a+b) e^{b\left(k x+r y+\frac{(k-r)^{3} b^{2} t^{\alpha}}{\alpha}+C\right)}-1}\right)
$$




$$
-\frac{(k-r)^{2}(a+b)^{2}}{2}\left(\frac{(a-b) e^{b\left(k x+r y+\frac{(k-r)^{3} b^{2} t^{\alpha}}{\alpha}+C\right)}-1}{(a+b) e^{b\left(k x+r y+\frac{(k-r)^{3} b^{2} t^{\alpha}}{\alpha}+C\right)}-1}\right)^{2} .
$$

By using Family 12, we get

$$
\begin{aligned}
u_{37}(x, y, t)= & \frac{(k-r)^{2}\left(b^{2}-c^{2}\right)}{2}-c(k-r)^{2}(b+c)\left(\frac{(b-c) e^{b\left(k x+r y+\frac{b^{2}(k-r)^{3} t^{\alpha}}{\alpha}+c\right)}-1}{(b+c) e^{b\left(k x+r y+\frac{b^{2}(k-r)^{3} t^{\alpha}}{\alpha}+c\right)}+1}\right) \\
& -\frac{(k-r)^{2}(b+c)^{2}}{2}\left(\frac{(b-c) e^{b\left(k x+r y+\frac{b^{2}(k-r)^{3} t^{\alpha}}{\alpha}+c\right)}-1}{(b+c) e^{b\left(k x+r y+\frac{b^{2}(k-r)^{3} t^{\alpha}}{\alpha}+c\right)}+1}\right)^{2} .
\end{aligned}
$$

By using Family 13, (23) becomes

$$
\begin{aligned}
u_{38}(x, y, t)= & \frac{(k-r)^{2}\left(b^{2}-c^{2}\right)}{2}+c(k-r)^{2}(b+c)\left(\frac{e^{b\left(k x+r y+\frac{(k-r)^{3} b^{2} t^{\alpha}}{\alpha}+c\right)}-b+c}{e^{b\left(k x+r y+\frac{(k-r)^{3} b^{2} t^{\alpha}}{\alpha}+c\right)}+b+c}\right) \\
& -\frac{(k-r)^{2}(b+c)^{2}}{2}\left(\frac{e^{b\left(k x+r y+\frac{(k-r)^{3} b^{2} t^{\alpha}}{\alpha}+C\right)}-b+c}{e^{b\left(k x+r y+\frac{(k-r)^{3} b^{2} t^{\alpha}}{\alpha}+C\right)}+b+c}\right)^{2} .
\end{aligned}
$$

By using Family 15, (23) can be written as

$$
u_{39}(x, y, t)=-\frac{(k-r)^{2} c^{2}}{2}+\frac{c^{3}(k-r)^{2}(k x+r y+C)}{c(k x+r y+C)+2}-\frac{(k-r)^{2} c^{4}(k x+r y+C)^{2}}{2(c(k x+r y+C)+2)^{2}} .
$$

By using Family 16, (23) becomes

$$
u_{40}(x, y, t)=-2 \frac{(k-r)^{2}}{(k x+r y+C)^{2}}
$$

By using Family 18, (23) reads

$$
u_{41}(x, y, t)=-\frac{(k-r)^{2} c^{2}}{2}-\frac{(k-r)^{2} c^{2}}{2}\left(\cot \left(\frac{c}{2}\left(k x+r y-\frac{(k-r)^{3} c^{2} t^{\alpha}}{\alpha}\right)+\frac{c}{2}\right)\right)^{2} .
$$

By using Family 19, one constructs for (23)

$$
u_{42}(x, y, t)=-2 a^{2}(k-r)^{2} c\left(e^{a\left(k x+r y+\frac{(k-r)^{3} a^{2} t^{\alpha}}{\alpha}+c\right)}-c\right)^{-1}-2(k-r)^{2} c^{2} a^{2}\left(e^{a\left(k x+r y+\frac{(k-r)^{3} a^{2} t^{\alpha}}{\alpha}+c\right)}-c\right)^{-2} .
$$

SET 5:

We have yielded the arbitrary constants as

$$
\left\{\begin{array}{c}
A_{0}=-\frac{(k-r)^{2}(b-c)(b+c)}{3}, A_{1}=0, A_{2}=-\frac{(k-r)^{2}(b-c)^{2}}{2}, B_{1}=0 \\
B_{2}=-\frac{(k-r)^{2}(b+c)^{2}}{2}, a=0, b=b, c=c, k=k, r=r, w=4(k-r)^{3}(b-c)(b+c)
\end{array}\right\}
$$

By using Family 1, (23) becomes

$$
\begin{aligned}
u_{43}(x, y, t)= & \frac{(k-r)^{2}(b-c)^{2}(b+c)^{2}}{3\left(-b^{2}+c^{2}\right)} \\
& -\frac{(k-r)^{2}(b-c)^{2}(b+c)^{2}}{2\left(-b^{2}+c^{2}\right)}\left(\tan \left(\frac{\sqrt{-b^{2}+c^{2}}}{2}\left(k x+r y-4 \frac{(k-r)^{3}(b-c)(b+c) t^{\alpha}}{\alpha}+C\right)\right)\right)^{2} \\
& -\frac{(k-r)^{2}(b-c)^{2}(b+c)^{2}}{2\left(-b^{2}+c^{2}\right)}\left(\tan \left(\frac{\sqrt{-b^{2}+c^{2}}}{2}\left(k x+r y-4 \frac{(k-r)^{3}(b-c)(b+c) t^{\alpha}}{\alpha}+C\right)\right)\right)^{-2} .
\end{aligned}
$$

By using Family 2, (23) can be written as 


$$
\begin{aligned}
u_{44}(x, y, t)= & -\frac{(k-r)^{2}\left(-4 b^{2} c^{2}+2 b^{4}+2 c^{4}\right)}{6\left(b^{2}-c^{2}\right)} \\
& -\frac{(k-r)^{2}\left(-6 b^{2} c^{2}+3 b^{4}+3 c^{4}\right)}{6\left(b^{2}-c^{2}\right)}\left(\tanh \left(\frac{\sqrt{b^{2}-c^{2}}}{2}\left(k x+r y-4 \frac{(k-r)^{3}(b-c)(b+c) t^{\alpha}}{\alpha}+C\right)\right)\right)^{2} \\
& -\frac{(k-r)^{2}\left(-6 b^{2} c^{2}+3 b^{4}+3 c^{4}\right)}{6\left(b^{2}-c^{2}\right)}\left(\tanh \left(\frac{\sqrt{b^{2}-c^{2}}}{2}\left(k x+r y-4 \frac{(k-r)^{3}(b-c)(b+c) t^{\alpha}}{\alpha}+C\right)\right)\right)^{-2} .
\end{aligned}
$$

By using Family 8, (23) reads

$$
u_{45}(x, y, t)=-2 \frac{(k-r)^{2}}{(k x+r y+C)^{2}} .
$$

By using Family 11, one constructs for (23)

$$
u_{46}(x, y, t)=-\frac{2(k-r)^{2}}{3} \frac{2 b^{2}+8 b^{4} e^{2 b\left(k x+r y-4 \frac{(k-r)^{3} b^{2} t^{\alpha}}{\alpha}+C\right)}+2 b^{6} e^{4 b\left(k x+r y-4 \frac{(k-r)^{3} b^{2} t^{\alpha}}{\alpha}+C\right)}}{\left(-1-e^{b\left(k x+r y-4 \frac{(k-r)^{3} b^{2} t^{\alpha}}{\alpha}+C\right)} b\right)^{2}\left(-1+e^{b\left(k x+r y-4 \frac{(k-r)^{3} b^{2} t^{\alpha}}{\alpha}+C\right)} b\right)^{2}} .
$$

By using Family 12, (23) becomes

$$
u_{47}(x, y, t)=-\frac{2(k-r)^{2}}{3} \frac{\left(\begin{array}{c}
c^{2}+2 b^{2}+\left(4 c^{3}-4 b^{2} c\right) e^{F}+\left(6 c^{4}-14 b^{2} c^{2}+8 b^{4}\right) e^{2 F} \\
+\left(4 c^{5}+4 c b^{4}-8 c^{3} b^{2}\right) e^{3 F}+\left(c^{6}-3 b^{4} c^{2}+2 b^{6}\right) e^{4 F}
\end{array}\right)}{\left(-1+(b-c) e^{F}\right)^{2}\left(1+(b+c) e^{F}\right)^{2}}
$$

where

$$
F=b\left(k x+r y-4 \frac{(k-r)^{3}(b-c)(b+c) t^{\alpha}}{\alpha}+C\right) .
$$

By using Family 13, (23) can be written as

$$
u_{48}(x, y, t)=\frac{2(k-r)^{2}}{3} \frac{\left(\begin{array}{c}
-12 a b^{4} e^{F}+\left(-8 b^{4}-10 a^{2} b^{2}\right) e^{2 F} \\
-12 a b^{2} e^{3 F}+\left(-2 b^{2}-a^{2}\right) e^{4 F}-2 b^{6}-b^{4} a^{2}
\end{array}\right)}{\left(e^{F}-b\right)^{2}\left(e^{F}+b\right)^{2}}
$$

where

$$
F=b\left(k x+r y-4 \frac{(k-r)^{3}(b+a)(b-a) t^{\alpha}}{\alpha}+C\right) .
$$

By using Family 16, (23) becomes

$$
u_{49}(x, y, t)=-2 \frac{(k-r)^{2}}{(k x+r y+C)^{2}} .
$$

SET 6:

We have yielded the arbitrary constants as

$$
\left\{\begin{array}{c}
A_{0}=(k-r)^{2}(b-c)(b+c), A_{1}=0, A_{2}=-\frac{(k-r)^{2}(b-c)^{2}}{2}, B_{1}=0, \\
B_{2}=-\frac{(k-r)^{2}(b+c)^{2}}{2}, a=0, b=b, c=c, k=k, r=r, w=-4(k-r)^{3}(b-c)(b+c)
\end{array}\right\}
$$

By using Family 1, (23) reads

$$
\begin{aligned}
u_{50}(x, y, t)= & (k-r)^{2}(b-c)(b+c)-\frac{(k-r)^{2}\left(-b^{2}+c^{2}\right)}{2}\left(\tan \left(\frac{\sqrt{-b^{2}+c^{2}}}{2}\left(k x+r y+4 \frac{(k-r)^{3}(b-c)(b+c) t^{\alpha}}{\alpha}+C\right)\right)\right)^{2} \\
& -\frac{(k-r)^{2}(b+c)^{2}(b-c)^{2}}{2\left(-b^{2}+c^{2}\right)\left(\tan \left(\frac{\sqrt{-b^{2}+c^{2}}}{2}\left(k x+r y+4 \frac{(k-r)^{3}(b-c)(b+c) t^{\alpha}}{\alpha}+C\right)\right)\right)^{2}}
\end{aligned}
$$


By using Family 2, we get

$$
\begin{aligned}
u_{51}(x, y, t)= & (k-r)^{2}(b-c)(b+c)-\frac{(k-r)^{2}\left(b^{2}-c^{2}\right)}{2}\left(\tanh \left(\frac{\sqrt{b^{2}-c^{2}}}{2}\left(k x+r y+4 \frac{(k-r)^{3}(b-c)(b+c) t^{\alpha}}{\alpha}+C\right)\right)\right)^{2} \\
& -\frac{(k-r)^{2}(b+c)^{2}(b-c)^{2}}{2\left(b^{2}-c^{2}\right)\left(\tanh \left(1 / 2 \sqrt{b^{2}-c^{2}}\left(k x+r y+4 \frac{(k-r)^{3}(b-c)(b+c) t^{\alpha}}{\alpha}+C\right)\right)\right)^{2}} .
\end{aligned}
$$

By using Family 4, one constructs for (23)

$$
\begin{aligned}
u_{52}(x, y, t)= & -(k-r)^{2} c^{2}-\frac{(k-r)^{2} c^{2}}{2}\left(\tan \left(\frac{\sqrt{c^{2}}}{2}\left(k x+r y-4 \frac{(k-r)^{3} c^{2} t^{\alpha}}{\alpha}+C\right)\right)\right)^{2} \\
& -\frac{(k-r)^{2} c^{2}}{2\left(\tan \left(\frac{\sqrt{c^{2}}}{2}\left(k x+r y-4 \frac{(k-r)^{3} c^{2} t^{\alpha}}{\alpha}+C\right)\right)\right)^{2}} .
\end{aligned}
$$

By using Family 5, (23) reads

$$
\begin{aligned}
u_{53}(x, y, t)= & (k-r)^{2}(b-c)(b+c)-\frac{(k-r)^{2}(b-c)(b+c)}{2}\left(\tanh \left(\frac{\sqrt{b^{2}-c^{2}}}{2}\left(k x+r y+4 \frac{(k-r)^{3}(b-c)(b+c) t^{\alpha}}{\alpha}+C\right)\right)\right)^{2} \\
& -\frac{(k-r)^{2}(b-c)(b+c)}{2\left(\tanh \left(\frac{\sqrt{b^{2}-c^{2}}}{2}\left(k x+r y+4 \frac{(k-r)^{3}(b-c)(b+c) t^{\alpha}}{\alpha}+C\right)\right)\right)^{2}} .
\end{aligned}
$$

By using Family 8, (23) can be written as

$$
u_{54}(x, y, t)=-2 \frac{(k-r)^{2}}{(k x+r y+C)^{2}}
$$

By using Family 11, (23) becomes

$$
\begin{aligned}
u_{55}(x, y, t)= & (k-r)^{2} b^{2}-\frac{(k-r)^{2} b^{2}}{2}\left(\frac{-1+b e^{b\left(k x+r y+4 \frac{(k-r)^{3} b^{2} t^{\alpha}}{\alpha}+C\right)}}{-1-b e^{b\left(k x+r y+4 \frac{(k-r)}{b^{2} t^{\alpha} t^{\alpha}}+C\right)}}\right)^{2} \\
& -\frac{(k-r)^{2} b^{2}}{2}\left(\frac{-1-b e^{b\left(k x+r y+4 \frac{(k-r)^{3} b^{2} t^{\alpha}}{\alpha}+C\right)}}{-1+b e^{b\left(k x+r y+4 \frac{(k-r)^{3} b^{2} t^{\alpha}}{\alpha}+C\right)}}\right)^{2} .
\end{aligned}
$$

By using Family 12, one constructs for (23)

$$
u_{56}(x, y, t)=-8 b^{4}(k-r)^{2} e^{2 b\left(k x+r y+4 \frac{(k-r)^{3} b^{2} t^{\alpha}}{\alpha}+C\right)}\left(b e^{b\left(k x+r y+4 \frac{(k-r)^{3} b^{2} t^{\alpha}}{\alpha}+C\right)}-1\right)^{-2}\left(b e^{b\left(k x+r y+4 \frac{(k-r)^{3} b^{2} t^{\alpha}}{\alpha}+C\right)}+1\right)^{-2}
$$

By using Family 13, we get

$$
\begin{aligned}
u_{57}(x, y, t)= & (k-r)^{2} b^{2}-\frac{(k-r)^{2} b^{2}}{2}\left(e^{b\left(k x+r y+4 \frac{(k-r)^{3} b^{2} t^{\alpha}}{\alpha}+C\right)}+b\right)^{2}\left(e^{b\left(k x+r y+4 \frac{(k-r)^{3} b^{2} t^{\alpha}}{\alpha}+C\right)}-b\right)^{-2} \\
& -\frac{(k-r)^{2} b^{2}}{2}\left(e^{b\left(k x+r y+4 \frac{(k-r)^{3} b^{2} t^{\alpha}}{\alpha}+C\right)}-b\right)^{2}\left(e^{b\left(k x+r y+4 \frac{(k-r)^{3} b^{2} t^{\alpha}}{\alpha}+C\right)}+b\right)^{-2} .
\end{aligned}
$$

By using Family 17, (23) becomes

$$
u_{58}(x, y, t)=-2 \frac{(k-r)^{2}}{(k x+r y+C)^{2}}
$$

By using Family 18, (23) reads

$$
\begin{aligned}
u_{59}(x, y, t)= & -(k-r)^{2} c^{2}-\frac{(k-r)^{2} c^{2}}{2}\left(\tan \left(\frac{c}{2}\left(k x+r y-4 \frac{(k-r)^{3} c^{2} t^{\alpha}}{\alpha}\right)+\frac{C}{2}\right)\right)^{2} \\
& -\frac{(k-r)^{2} c^{2}}{2}\left(\cot \left(\frac{c}{2}\left(k x+r y-4 \frac{(k-r)^{3} c^{2} t^{\alpha}}{\alpha}\right)+\frac{c}{2}\right)\right)^{2} .
\end{aligned}
$$



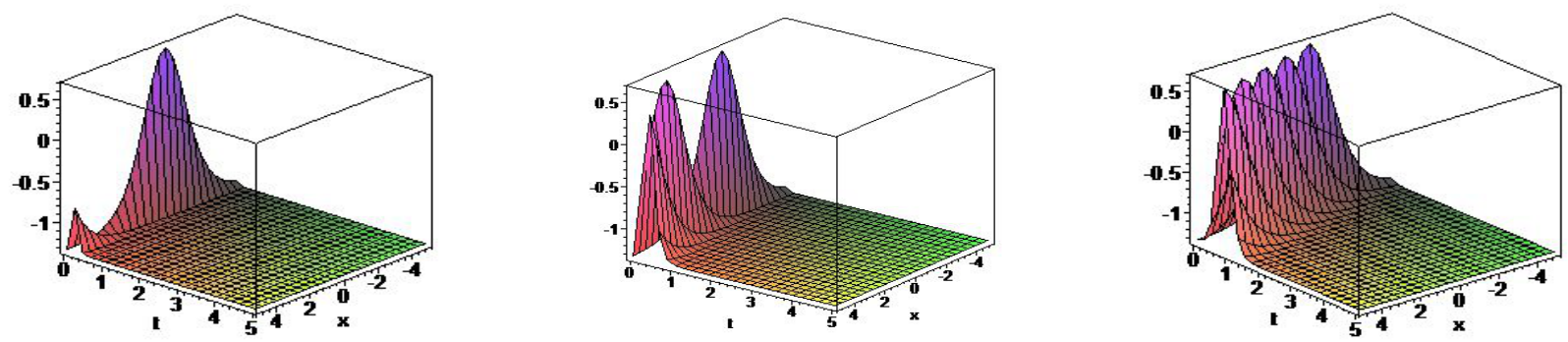

Figure 1: Graph of $u_{5}(x, y, t)$ of Eq. (28) corresponding to $b=0.4, r=2, k=-3, C=0, y=0$ for $\alpha=0.43, \alpha=0.61$ and $\alpha=0.96$, respectively.
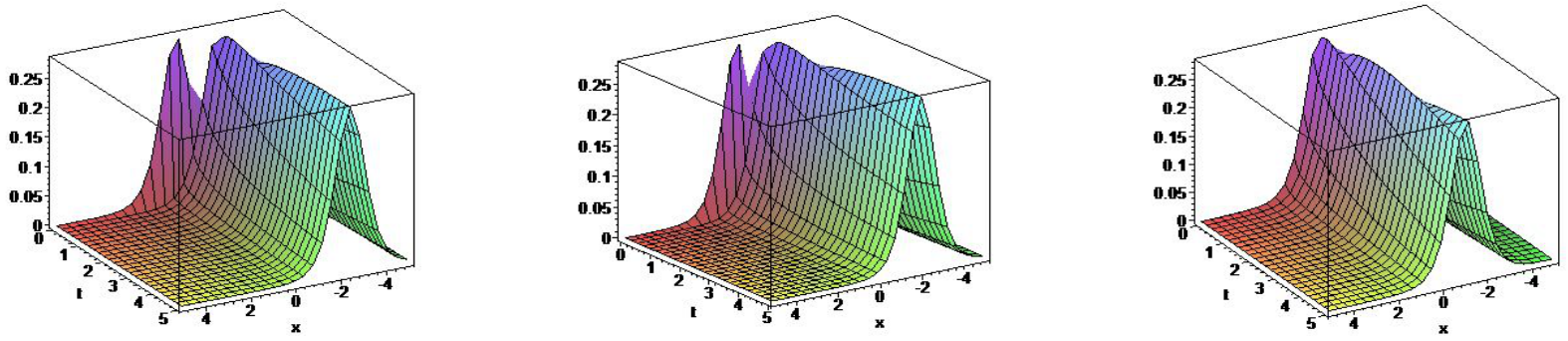

Figure 2: Graph of $u_{18}(x, y, t)$ of Eq. (41) corresponding to $A_{1}=0, c=-2, r=2, k=3, C=0, y=0$ for $\alpha=0.1, \alpha=0.13$ and $\alpha=0.5$, respectively.
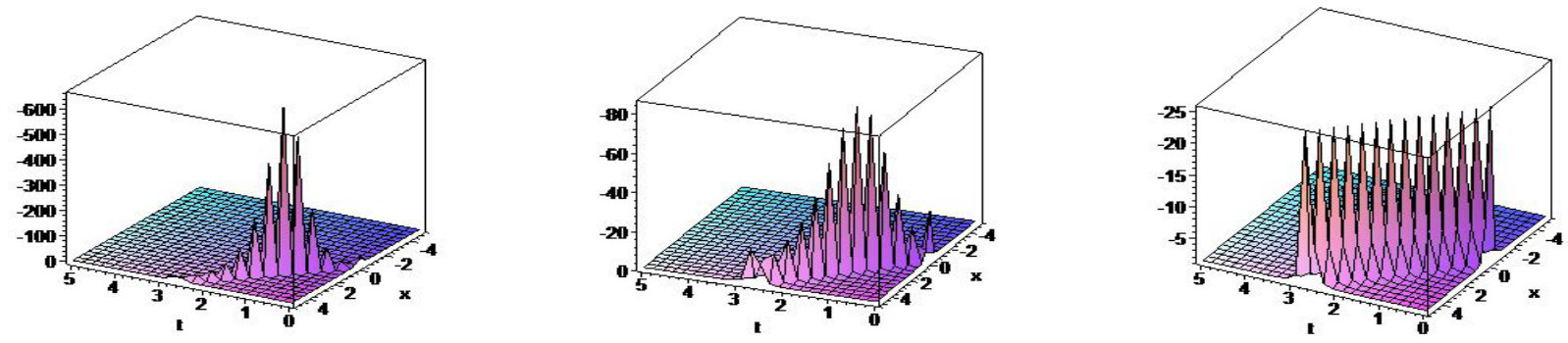

Figure 3: Graph of $u_{23}(x, y, t)$ of Eq. (46) corresponding to $a=1, b=2, c=1, r=1, k=2, C=0, y=0$ for $\alpha=0.89, \alpha=$ 0.91 and $\alpha=1$, respectively.
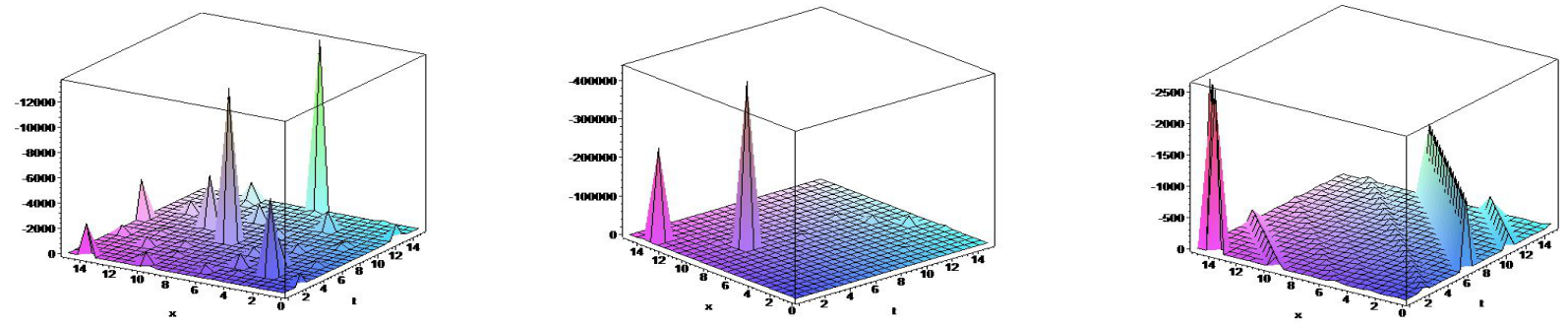

Figure 4: Graph of $u_{32}(x, y, t)$ of Eq. (55) corresponding to $a=1, b=1, c=2, r=1, k=2, C=0, y=0$ for $\alpha=0.1, \alpha=$ 0.5 and $\alpha=1$, respectively. 

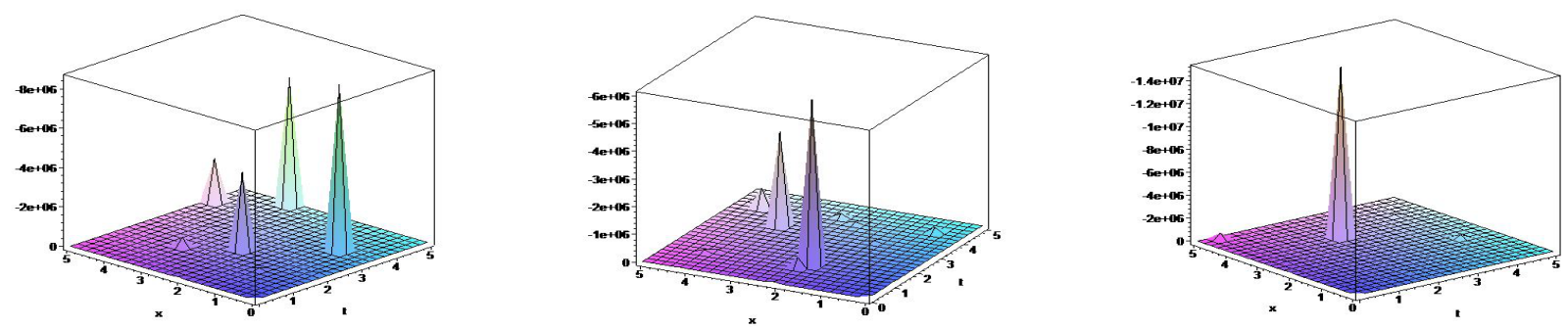

Figure 5: Graph of $u_{41}(x, y, t)$ of Eq. (56) corresponding to $a=0, b=0, c=1, r=-2, k=3, C=0, y=0$ for $\alpha=0.1, \alpha=0.55$ and $\alpha=1$, respectively.
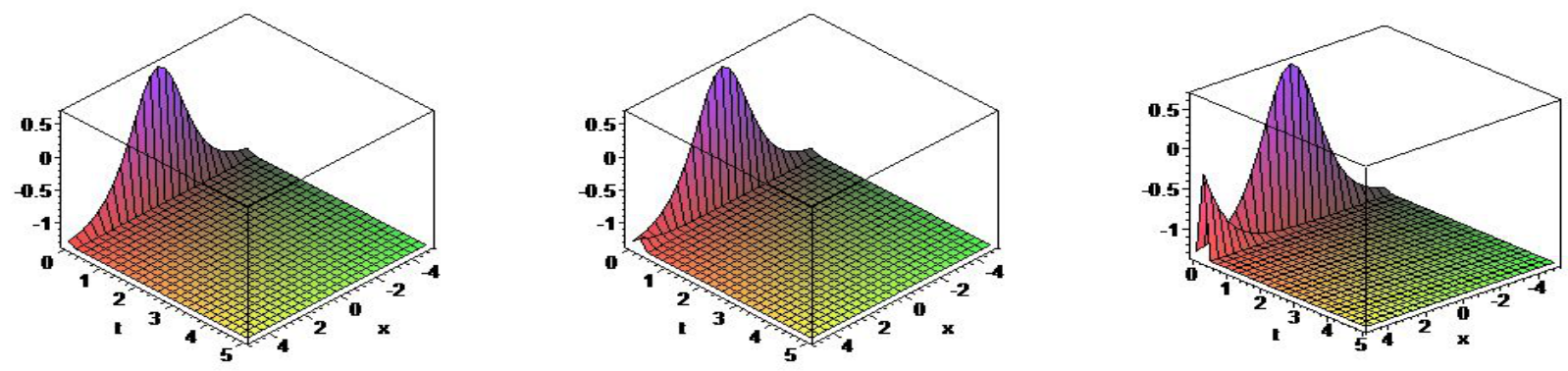

Figure 6: Graph of $u_{47}(x, y, t)$ of Eq. (60) corresponding to $b=1, c=0, r=1, k=-1, C=0, y=0$ for $\alpha=0.6, \alpha=0.89$ and $\alpha=0.999$, respectively.

\section{Application of Jacobi elliptic function expansion method to conformable (2+1) dimensional KdV equation}

To solve Eq. (3) by the Jacobian elliptic function expansion method, considering $u(x, t)=u(\xi), \xi=k x+r y-\frac{w}{\alpha} t^{\alpha}$ and integrating once with respect to $\xi$, Eq. (3) becomes

$$
-w u-(3 k-3 r) u^{2}-\left(k^{3}-r^{3}-3 r k^{2}+3 r^{2} k\right) u^{\prime \prime}=0 .
$$

The Solutions in Terms of $\operatorname{sn}(\xi)$ :

By balancing the highest-order linear term and the highest-order nonlinear term we obtain $n=2$, thus the solution of Eq. (63) can be expressed as

$$
u(\xi)=a_{0}+a_{1} \operatorname{sn}(\xi)+a_{2} s^{2}(\xi) .
$$

Substituting Eq. (64) into Eq. (63) and collecting various powers of $\operatorname{sn}(\xi)$, we get

$$
\begin{aligned}
& 18 k r^{2} a_{2} m^{2}+6 k^{3} a_{2} m^{2}-6 r^{3} a_{2} m^{2}-3 r a_{2}^{2}-18 k^{2} r a_{2} m^{2}+3 k a_{2}{ }^{2}=0, \\
& 2 k^{3} a_{1} m^{2}+6 k r^{2} a_{1} m^{2}-2 r^{3} a_{1} m^{2}-6 k^{2} r a_{1} m^{2}+6 k a_{1} a_{2}-6 r a_{1} a_{2}=0, \\
& w a_{2}+4 r^{3} a_{2} m^{2}+4 r^{3} a_{2}-4 k^{3} a_{2} m^{2}-12 k r^{2} a_{2} m^{2}-12 k r^{2} a_{2}-4 k^{3} a_{2} \\
& +6 k a_{0} a_{2}+3 k a_{1}^{2}+12 k^{2} r a_{2}-6 r a_{0} a_{2}+12 k^{2} r a_{2} m^{2}-3 r a_{1}^{2}=0, \\
& -3 k r^{2} a_{1} m^{2}+w a_{1}+3 k^{2} r a_{1} m^{2}+6 k a_{0} a_{1}-k^{3} a_{1} m^{2}+r^{3} a_{1} \\
& +3 k^{2} r a_{1}-k^{3} a_{1}+r^{3} a_{1} m^{2}-6 r a_{0} a_{1}-3 k r^{2} a_{1}=0 \\
& 2 k^{3} a_{2}+w a_{0}+6 k r^{2} a_{2}-2 r^{3} a_{2}-3 r a_{0}^{2}-6 k^{2} r a_{2}+3 k a_{0}^{2}=0 .
\end{aligned}
$$

By solving the above system of equations using any package of symbolic computations, we can determine the values of the coefficients as:

$$
a_{0}=4\left(\frac{m^{2}}{6}+\frac{1}{6} \pm \frac{1}{6} \sqrt{m^{4}-m^{2}+1}\right)(k-r)^{2},
$$




$$
\begin{aligned}
a_{1} & =0, a_{2}=-2\left(k^{2}-2 k r+r^{2}\right) m^{2}, \\
w & =\mp 4 \sqrt{m^{4}-m^{2}+1}(k-r)^{3} .
\end{aligned}
$$

Substituting Eq. (65) into Eq. (64), we obtain the exact solution of Eq. (3) in the form

$$
u(\xi)=4\left(\frac{m^{2}}{6}+\frac{1}{6} \pm \frac{1}{6} \sqrt{m^{4}-m^{2}+1}\right)(k-r)^{2}-2\left(k^{2}-2 k r+r^{2}\right) m^{2} s n^{2}(\xi \mid m) \text { with } \xi=k x+r y-\frac{w}{\alpha} t^{\alpha},
$$

which is an exact periodic solution of the KdV equation with conformable derivative. For $m \rightarrow 1, \operatorname{sn}(\xi \mid m) \rightarrow \tanh (\xi)$ and the above exact periodic solution is degenerated into a new form of solution can be written as

$$
u(x, y, t)=4\left(\frac{1}{3} \pm \frac{1}{6}\right)(k-r)^{2}-2\left(k^{2}-2 k r+r^{2}\right) \tanh ^{2}\left(k x+r y \pm \frac{4(k-r)^{3}}{\alpha} t^{\alpha}\right) .
$$
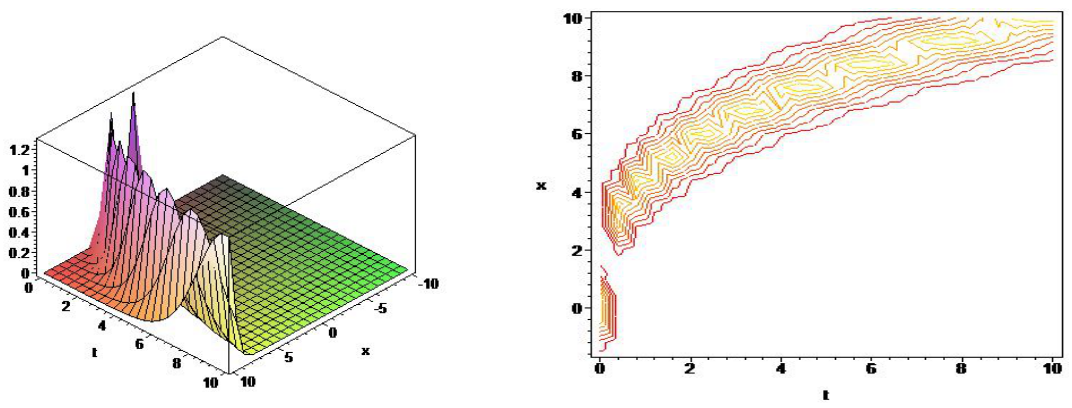

Figure 7: Graph of Eq. (67) corresponding to $k=1.3, r=2.1, y=0$ for $\alpha=0.35$.

\section{The Solutions in Terms of $c n(\xi)$ :}

By balancing the highest-order linear term and the highest-order nonlinear term we obtain $n=2$, thus the solution of Eq. (63) can be expressed by Jacobi elliptic cosine function

$$
u(\xi)=b_{0}+b_{1} c n(\xi)+b_{2} c n^{2}(\xi) .
$$

Substituting Eq. (68) into Eq. (63) and collecting various powers of $c n(\xi)$, we get an algebraic equations system by solving this system of equations using any package of symbolic computations, we can determine the values of the coefficients as:

$$
\begin{aligned}
b_{0} & =4\left(-\frac{m^{2}}{3}+\frac{1}{6} \pm \frac{1}{6} \sqrt{m^{4}-m^{2}+1}\right)(k-r)^{2}, \\
b_{1} & =0, b_{2}=2\left(k^{2}-2 k r+r^{2}\right) m^{2}, \\
w & =\mp 4 \sqrt{m^{4}-m^{2}+1}(k-r)^{3} .
\end{aligned}
$$

Substituting Eq. (69) into Eq. (68), we obtain the exact solution of Eq. (3) in the form

$$
u(\xi)=4\left(-\frac{m^{2}}{3}+\frac{1}{6} \pm \frac{1}{6} \sqrt{m^{4}-m^{2}+1}\right)(k-r)^{2}+2\left(k^{2}-2 k r+r^{2}\right) m^{2} c n^{2}(\xi \mid m) \text { with } \xi=k x+r y-\frac{w}{\alpha} t^{\alpha}
$$

which is an exact periodic solution of the KdV equation with conformable derivative. For $m \rightarrow 1, c n(\xi \mid m) \rightarrow \sec h(\xi)$ and the above exact periodic solution is degenerated into a new form of solution can be written as

$$
u(x, y, t)=4\left(-\frac{1}{6} \pm \frac{1}{6}\right)(k-r)^{2}+2\left(k^{2}-2 k r+r^{2}\right) \sec ^{2}\left(k x+r y \pm \frac{4(k-r)^{3}}{\alpha} t^{\alpha}\right) .
$$



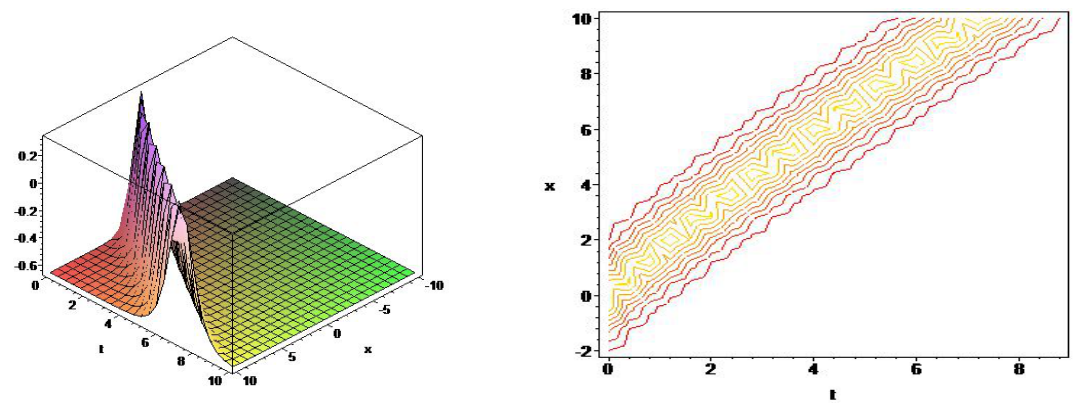

Figure 8: Graph of Eq. (71) corresponding to $k=0.9, r=1.6, y=0$ for $\alpha=0.9$.

\section{Numerical application of C-VIM to conformable $(2+1)$-dimensional KdV equation}

In this section, we consider the exact solution Eq. (32) (namely $\left.u_{9}(x, y, t)\right)$ of $(2+1)$-dimensional conformable derivative $\mathrm{KdV}$ equation (3) for special values of parameters $(c=2, k=2, r=1, C=0)$ subject to initial condition

$$
u(x, y, 0)=-\frac{2}{3}-2(\tan (2 x+y))^{2} .
$$

For $\alpha=1$, the exact solution of (3) is

$$
u(x, y, t)=-\frac{2}{3}-2(\tan (2 x+y+4 t))^{2} .
$$

Solution by C-VIM:

For solving Eq. (3) numerically corresponding to the initial condition (72) by C-VIM, we obtain the recurrence relation

$$
\begin{aligned}
u_{n+1}(x, y)= & u_{n}(x, y, t)+\int_{0}^{t} \lambda(\zeta)\left[\zeta^{1-\alpha} \frac{\partial u_{n}(x, y, \zeta)}{\partial \zeta}--6 \frac{\partial u_{n}(x, y, \zeta)}{\partial x} u_{n}(x, y, \zeta)+6 \frac{\partial u_{n}(x, y, \zeta)}{\partial y} u_{n}(x, y, \zeta)-\frac{\partial^{3} u_{n}(x, y, \zeta)}{\partial x^{3}}\right. \\
& \left.+\frac{\partial^{3} u_{n}(x, y, \zeta)}{\partial y^{3}}+3 \frac{\partial^{3} u_{n}(x, y, \zeta)}{\partial x^{2} \partial y}-3 \frac{\partial^{3} u_{n}(x, y, \zeta)}{\partial x \partial y^{2}}\right] d \zeta
\end{aligned}
$$

and following conditions:

$$
\left.\lambda^{\prime}(\zeta)\right|_{\zeta=t}=0,\left.\quad \lambda(\zeta)\right|_{\zeta=t}=-1 .
$$

Therefore, the Lagrange multiplier can be identified as $\lambda=-1$. As a result we obtain the following iteration formula:

$$
\begin{aligned}
u_{n+1}(x, y)= & u_{n}(x, y, t)-\int_{0}^{t}\left[\zeta^{1-\alpha} \frac{\partial u_{n}(x, y, \zeta)}{\partial \zeta}--6 \frac{\partial u_{n}(x, y, \zeta)}{\partial x} u_{n}(x, y, \zeta)+6 \frac{\partial u_{n}(x, y, \zeta)}{\partial y} u_{n}(x, y, \zeta)-\frac{\partial^{3} u_{n}(x, y, \zeta)}{\partial x^{3}}\right. \\
& \left.+\frac{\partial^{3} u_{n}(x, y, \zeta)}{\partial y^{3}}+3 \frac{\partial^{3} u_{n}(x, y, \zeta)}{\partial x^{2} \partial y}-3 \frac{\partial^{3} u_{n}(x, y, \zeta)}{\partial x \partial y^{2}}\right] d \zeta
\end{aligned}
$$

By the initial condition (72) we write

$$
u_{0}(x, y, t)=u(x, y, 0)=-\frac{2}{3}-2(\tan (2 x+y))^{2} .
$$

Now, substituting (75) into (74) and then iterate for $n=0, n=1$, and $n=2$ respectively, we yield

$$
\begin{array}{r}
u_{1}(x, y, t)=-\frac{2}{3}-2(\tan (2 x+y))^{2}-\frac{16 \sin (2 x+y) t}{(\cos (2 x+y))^{3}}, \\
u_{2}(x, y, t)=-\frac{2}{3}-2(\tan (2 x+y))^{2}-\frac{32 \sin (2 x+y) t}{(\cos (2 x+y))^{3}}
\end{array}
$$




$$
\begin{aligned}
& +\left(-\frac{16 t^{2-\alpha}}{(-2+\alpha)(\cos (2 x+y))^{3}}-\frac{1024 t^{3}}{(\cos (2 x+y))^{5}}+\frac{1536 t^{3}}{(\cos (2 x+y))^{7}}\right) \sin (2 x+y) \\
& +\frac{64 t^{2}}{(\cos (2 x+y))^{2}}-\frac{96 t^{2}}{(\cos (2 x+y))^{4}} \cdot \\
u_{3}(x, y, t)= & \left(\frac{64 t-16 t^{2-\alpha}-32 t \alpha}{(-2+\alpha)} A^{3}-1024 t^{3} A^{5}+1536 t^{3} A^{7}\right) B \\
+ & \frac{4}{3}+\left(64 t^{2}-2\right) A^{2}-96 t^{2} A^{4}+14155776 t^{7} B A^{15}-28311552 t^{7} B A^{13}-1622016 t^{6} A^{12} \\
+ & \left(\frac{196608 t^{5}\left(-21+640 t^{2}\right)}{7}+\frac{1474560 t^{6-\alpha}}{(-2+\alpha)(-6+\alpha)}\right) B A^{11} \\
+ & 50688 t^{4}\left(64 t^{2}+3\right) A^{10}-\left(\frac{24576 t^{5}\left(-1183+5120 t^{2}\right)}{35}+\frac{1966080 t^{6-\alpha}}{(-2+\alpha)(-6+\alpha)}\right) B A^{9} \\
& -\left(512 t^{4}\left(411+3968 t^{2}\right)+64512 \frac{t^{5-\alpha}}{(-5+\alpha)(-2+\alpha)}\right) A^{8} \\
& -\left(\frac{4608 t^{3}\left(-5+304 t^{2}\right)}{5}+\frac{4608 t^{5-2 \alpha}}{(-5+2 \alpha)(-2+\alpha)^{2}}-\frac{589824 t^{6-\alpha}}{(-2+\alpha)(-6+\alpha)}-\frac{4608(-6+\alpha) t^{4-\alpha}}{(-4+\alpha)(-2+\alpha)}\right) B A^{7} \\
+ & \left(2048 t^{4}\left(29+192 t^{2}\right)+86016 \frac{t^{5-\alpha}}{(-5+\alpha)(-2+\alpha)}\right) A^{6} \\
+ & \left(\frac{512 t^{3}\left(-35+96 t^{2}\right)}{5}-\frac{3072(-6+\alpha) t^{4-\alpha}}{(-4+\alpha)(-2+\alpha)}+\frac{3072 t^{5-2 \alpha}}{(-5+2 \alpha)(-2+\alpha)^{2}}\right) B A^{5} \\
+ & +\left(\frac{16 t\left(-3+32 t^{2}\right)}{3}+\frac{16 t^{-2 \alpha+3}}{2 \alpha-3}-\frac{32 t^{2-\alpha}}{-2+\alpha}\right) B A^{3} \\
+ & \left(-2+\alpha t^{2}\left(-3+64 t^{2}\right)-192 \frac{t^{3-\alpha}}{-2+\alpha}-24576 \frac{t^{5-\alpha}}{(-5+\alpha)(-2+\alpha)}\right) A^{4} \\
& \left(\frac{12+\alpha}{2-\alpha} A^{2}\right.
\end{aligned}
$$

where $A^{-1}=\cos (2 x+y), B=\sin (2 x+y)$.

Remark 1. The accuracy of all the results obtained in this study was provided by using maple.

\section{Conclusion}

In this work, we investigated the $(2+1)$-dimensional conformable derivative KdV equation for which we constructed exact wave solutions with the help of ITEM and Jacobi elliptic function expansion method. ITEM is advantageous in terms of simplicity and diversity of solutions, the other method is advantageous in that it obtains the results directly, quickly and needs simple algorithms. Traveling wave solutions including soliton, periodic, kink and kink singular wave solutions of the model studied using the presented methods were found. Based on the ITEM devised, we have consructed dark soliton solutions such as $u_{2}, u_{3}, u_{12}, \ldots$, exponential solutions such as $u_{5}, u_{7}, u_{15}, u_{31}, \ldots$, trigonometric soliton solutions such as $u_{9}, u_{11}, u_{22}, \ldots$ and rational solutions such as $u_{4}, u_{8}, u_{28}, \ldots$. Dark $((67))$ and bright ((71)) soliton solutions were also obtained as a result of the application of the Jacobi elliptic function expansion method. We use different values of coefficients for obtaining novel graphical representations of solutions which are helpful for researchers to understand the physical phenomena of underlying model.

In Figs. 1-6, we plot three dimensional graphics of $u_{5}, u_{18}, u_{23}, u_{32}, u_{41}$ and $u_{47}$, which denote the dynamics of solutions with appropriate parametric selections. These graphics were drawn according to different values of $\alpha$, which is the order of fractional derivative, and it is tried to increase the intelligibility of dynamic behavior while changing $\alpha$. 
Table 1: Comparison of obtained result by 3-th iteration and exact solution (32) of (3).

\begin{tabular}{ccc|ccc}
\hline \multicolumn{5}{c}{$\alpha=1.0$} \\
\hline$x$ & $y$ & $t$ & $C-$ VIM & Exact & Error \\
\hline 0.001 & 0.001 & 0.001 & -0.6667650 & -0.6667647 & $-0.3 \times 10^{-6}$ \\
& & 0.002 & -0.6669090 & -0.6669087 & $-0.3 \times 10^{-6}$ \\
0.01 & 0.01 & 0.01 & -0.6764517 & -0.6764988 & $0.471 \times 10^{-4}$ \\
& & 0.02 & -0.6903011 & -0.6910632 & $0.7621 \times 10^{-3}$ \\
& & 0.03 & -0.7084940 & -0.7123504 & $0.38564 \times 10^{-2}$ \\
& & 0.04 & -0.7284763 & -0.7406405 & $0.121642 \times 10^{-1}$ \\
0.02 & 0.02 & 0.05 & -0.7466970 & -0.7763130 & $0.296160 \times 10^{-1}$ \\
& & 0.02 & -0.6867516 & -0.6868008 & $0.492 \times 10^{-4}$ \\
& & 0.03 & -0.7288268 & -0.7328925 & $0.40657 \times 10^{-2}$ \\
& & 0.04 & -0.7538605 & -0.7666780 & $0.128175 \times 10^{-1}$ \\
& & 0.05 & -0.7770070 & -0.8082016 & $0.311946 \times 10^{-1}$ \\
0.01 & 0.02 & 0.03 & -0.7148429 & -0.7187534 & $0.39105 \times 10^{-2}$ \\
& & 0.04 & -0.7365185 & -0.7488494 & $0.123309 \times 10^{-1}$ \\
0.02 & 0.01 & 0.03 & -0.7216186 & -0.7255988 & $0.39802 \times 10^{-2}$ \\
& & 0.04 & -0.7449777 & -0.7575261 & $0.125484 \times 10^{-1}$ \\
\hline
\end{tabular}

Table 2: Comparison of obtained result (32) of (3) by 3-th iteration for different $\alpha$ values.

\begin{tabular}{|c|c|c|c|c|}
\hline & & & $\alpha=0.8$ & $\alpha=0.9$ \\
\hline$\chi$ & $y$ & $t$ & $C-V I M$ & $C-V I M$ \\
\hline \multirow[t]{5}{*}{0.01} & 0.01 & 0.01 & -0.6860734 & -0.6821012 \\
\hline & & 0.02 & -0.7155144 & -0.7051462 \\
\hline & & 0.03 & -0.7546394 & -0.7356537 \\
\hline & & 0.04 & -0.8001101 & -0.7705637 \\
\hline & & 0.05 & -0.8476016 & -0.8058438 \\
\hline \multirow[t]{5}{*}{0.02} & 0.02 & 0.01 & -0.7017910 & -0.6953636 \\
\hline & & 0.02 & -0.7404156 & -0.7255605 \\
\hline & & 0.03 & -0.7880061 & -0.7628366 \\
\hline & & 0.04 & -0.8411520 & -0.8040732 \\
\hline & & 0.05 & -0.8953551 & -0.8450957 \\
\hline
\end{tabular}

Solution $u_{32}$ represent the soliton wave solution which are very important and remarkable as there are waves that can protect their identity in interaction with other waves. Solutions $u_{23}$ and $u_{41}$ represent the exact periodic wave solution. Solution $u_{5}, u_{18}$ and $u_{47}$ represent the singular kink-type traveling wave solutions. In Figs. 7 and 8 , under the choice of the suitable values of parameters, the 3D the contour graphs are plotted. Some obtained solutions behave as periodic traveling waves, bright soliton, and dark soliton solutions. When the modulus $m \rightarrow 1$ some of these obtained solutions degenerate as solitary wave solutions. Moreover, we applied the conformable variational iteration method by using of the exact solution (32) of $(2+1)$-dimensional conformable KdV equation. For the appropriate values of $x, y, t$ and different values of $\alpha(\alpha=0.8, \alpha=0.9, \alpha=1.0)$, we compared the exact solution and iterated solution in Table 1 and 2 . We hope this study will guide future research and be useful for engineers and scientists in this field. 
Funding information: The authors state no funding involved.

Author contributions: All authors have accepted responsibility for the entire content of this manuscript and approved its submission.

Conflict of interest: The authors state no conflict of interest.

\section{References}

[1] Ali U, Kamal R, Mohyud-Din ST. On nonlinear fractional differential equations. Int J Mod Math Sci. 2012;3(3).

[2] Podlubny I. Fractional differential equations. vol. 198 of Mathematics in Science and Engineering. 1999.

[3] Caputo M, Fabrizio M. A new definition of fractional derivative without singular kernel. Progr Fract Differ Appl. 2015;1(2):7385.

[4] Atangana A, Koca I. Chaos in a simple nonlinear system with Atangana-Baleanu derivatives with fractional order. Chaos Soliton Fract. 2016;89:447-454.

[5] Atangana A, Baleanu D. New fractional derivatives with nonlocal and non-singular kernel: theory and application to heat transfer model. Therm Sci 2016. OnLine-First (00). 18. 10.2298/TSCl160111018A.

[6] Atangana A, Baleanu D, Alsaedi A. New properties of conformable derivative. Open Mathematics. 2015;13(1).

[7] Khalil R, Al Horani M, Yousef A, Sababheh M. A new definition of fractional derivative. J Comput Appl Math. 2014;264,65-70.

[8] Abdeljawad T. On conformable fractional calculus. J Comput Appl Math. 2015;279:57-66.

[9] Yel G, Sulaiman TA, Baskonus H. On the complex solutions to the (3+1)-dimensional conformable fractional modified KdV-Zakharov-Kuznetsov equation. Mod Phys Lett B. 2020;2050069.

[10] Ravichandran C, Jothimani K, Baskonus HM, Valliammal N. New results on nondensely characterized integrodifferential equations with fractional order. Eur Phys J Plus. 2018;133(3), 109.

[11] Ekici M, Mirzazadeh M, Eslami M, Zhou Q, Moshokoa SP, Biswas A, Belic M. Optical soliton perturbation with fractionaltemporal evolution by first integral method with conformable fractional derivatives. Optik. 2016;127(22):10659-10669.

[12] Yavari M, Nazemi A. On fractional infinite-horizon optimal control problems with a combination of conformable and CaputoFabrizio fractional derivatives. ISA transactions.2020;101:7890.

[13] Bulut H, Sulaiman TA, Baskonus HM. Dark, bright optical and other solitons with conformable space-time fractional secondorder spatiotemporal dispersion. Optik. 2018;163:1-7.

[14] Rezazadeh H, Osman MS, Eslami M, Mirzazadeh M, Zhou Q, Badri SA, Korkmaz A. Hyperbolic rational solutions to a variety of conformable fractional Boussinesq-Like equations. Nonlinear Engineering. 2019;8(1):224-230.
[15] Zafar A. Rational exponential solutions of conformable spacetime fractional equal-width equations. Nonlinear Engineering. 2019;8(1):350-355.

[16] Park C, Nuruddeen RI, Ali KK, Muhammad L, Osman MS, Baleanu D. Novel hyperbolic and exponential ansatz methods to the fractional fifth-order Korteweg-de Vries equations. Adv Differ Equ. 2020;(1):1-12.

[17] Eslami M, Vajargah BF, Mirzazadeh M, Biswas A. Application of first integral method to fractional partial differential equations. Indian J Phys. 2014;88(2):177-184.

[18] Eslami M, Rezazadeh H. The first integral method for WuZhang system with conformable time-fractional derivative. Calcolo. 2016; 53(3):475-485.

[19] Ekici M, Mirzazadeh M, Eslami M, Zhou Q, Moshokoa SP, Biswas A, Belic M. Optical soliton perturbation with fractionaltemporal evolution by first integral method with conformable fractional derivatives. Optik. 2016;127(22):10659-10669.

[20] Bulut H, Baskonus HM, Pandir Y. The modified trial equation method for fractional wave equation and time fractional generalized Burgers equation. Abstr Appl Anal(Vol. 2013). Hindawi.2013.

[21] Bulut H, Pandir Y. Modified trial equation method to the nonlinear fractional Sharma-Tasso-Olever equation. Int I Model Optim. 2013;3(4):353.

[22] Aslan I. Exact solutions for fractional DDEs via auxiliary equation method coupled with the fractional complex transform. Math Methods Appl Sci. 2016;39(18):5619-5625.

[23] Akbulut A, Kaplan M. Auxiliary equation method for timefractional differential equations with conformable derivative. Comput Math with Appl. 2018;75(3):876-882.

[24] Hosseini K, Bekir A, Ansari R. New exact solutions of the conformable time-fractional Cahn-Allen and Cahn-Hilliard equations using the modified Kudryashov method. Optik. 2017;132:203-209.

[25] Hosseini K, Ansari R. New exact solutions of nonlinear conformable time-fractional Boussinesq equations using the modified Kudryashov method. Waves in Random and Complex Media. 2017;27(4): 628-636.

[26] Kumar D, Seadawy AR, Joardar AK. Modified Kudryashov method via new exact solutions for some conformable fractional differential equations arising in mathematical biology. Chin J Phys. 2018;56(1):75-85.

[27] Tasbozan O, Çenesiz Y, Kurt A. New solutions for conformable fractional Boussinesq and combined KdV-mKdV equations using Jacobi elliptic function expansion method. Eur Phys J Plus. 2016;131(7):244.

[28] Prakash A, Goyal M, Gupta S. Fractional variational iteration method for solving time-fractional Newell-Whitehead-Segel equation. Nonl Eng. 2019;8(1):164-171.

[29] Liang J, Tang L, Xia Y, Zhang Y. Bifurcations and exact solutions for a class of MKdV equations with the conformable fractional derivative via dynamical system method. Int J Bifurcat Chaos. 2020;30(01):2050004.

[30] Khater MM, Ghanbari B, Nisar KS, Kumar D. . Novel exact solutions of the fractional Bogoyavlensky-Konopelchenko equation involving the Atangana-Baleanu-Riemann derivative. Alex Eng J. 2020;59(5):2957-2967.

[31] Akgul A, Modanli M. Crank-Nicholson difference method and reproducing kernel function for third order fractional differential equations in the sense of Atangana-Baleanu Caputo 
derivative. Chaos Soliton Fract. 2019;127:10-16.

[32] Zabusky NJ. A synergetic approach to problems of nonlinear dispersive wave propagation and interaction. In Nonlinear partial differential equations. Academic Press. 1967:223-258.

[33] Karakoc SB, Ali KK. New Exact Solutions and Numerical Approximations of the Generalized KdV Equation. Comput Methods Differ Equ. 2020.

[34] Sarboland M, Aminataei A. On the numerical solution of the nonlinear Korteweg-de Vries equation. Syst Sci Control Eng. 2015;3(1):69-80.

[35] Gardner CS, Morikawa GK. The effect of temperature on the width of a small-amplitude, solitary wave in a collision-free plasma. Commun Pur Appl Math. 1965;18(1-2):35-49.

[36] Washimi H, Taniuti T. Propogation of ion acoustic solitary waves of small amplitude. Phys Rev Lett. 1966;17: 996-998.

[37] Dodd RK, Morris HC, Eilbeck JC, Gibbon JD. Soliton and nonlinear wave equations. London and New York, Academic Press. 1982;1982:640 p.

[38] Wazwaz AM. Two new Painlevé-integrable (2+1) and (3+ 1)dimensional KdV equations with constant and time-dependent coefficients. Nucl Phys B. 2020;115009.

[39] Liu H, Bai CL, Xin X, Li X. Equivalent transformations and exact solutions to the generalized cylindrical KdV type of equation. Nucl Phys B. 2020;114924.

[40] ter Braak F, Ferreira LA, Zakrzewski WJ. Quasi-integrability of deformations of the KdV equation. Nucl Phys B. 2019; 939;4994.

[41] Adamopoulou P, Papamikos G. Drinfel'd-Sokolov construction and exact solutions of vector modified KdV hierarchy. Nucl Phys B. 2020;952:114933.

[42] Ablowitz MJ, Segur H. Solitons and the inverse scattering transform (Vol. 4). SiaM 1981.

[43] Hirota R. The direct method in soliton theory (Vol. 155). Cambridge University Press. 2004.

[44] Wang G, Kara AH, Fakhar K, Vega-Guzman J, Biswas A. Group analysis, exact solutions and conservation laws of a generalized fifth order KdV equation. Chaos Soliton Fract. 2016;86:815.

[45] Geng X, Xue B. N-soliton and quasi-periodic solutions of the KdV6 equations. Appl Math Comput. 2012;219(8):3504-3510.

[46] Wang G, Kara AH. A (2+1)-dimensional KdV equation and mKdV equation: Symmetries, group invariant solutions and conservation laws. Phys Lett A. 2019;383(8):728-731.

[47] Elmandouha AA, Ibrahim AG. Bifurcation and travelling wave solutions for a (2+1)-dimensional KdV equation. J Taibah Univ Sci. 2020;14(1):139-147.

[48] Liu JG, Zhu WH, Zhou L, Xiong YK. Multi-waves, breather wave and lump-stripe interaction solutions in a (2+1)-dimensional variable-coefficient Korteweg-de Vries equation. Nonlinear Dyn. 2019;97(4):2127-2134.

[49] Adem AR. On the solutions and conservation laws of a twodimensional Korteweg de Vries model: Multiple exp-function method. J Appl Anal. 2018;24(1):27-33.

[50] Boiti M, Leon JP, Manna M, Pempinelli F. On the spectral transform of a Korteweg-de Vries equation in two spatial dimensions. Inverse problems. 1986;2(3):271.

[51] Manafian J, Lakestani M, Bekir A. Study of the analytical treatment of the (2+1)-dimensional Zoomeron, the Duffing and the SRLW equations via a new analytical approach. Int J Appl Comput Math. 2016;2(2):243-268.
[52] Ma HC, Zhang ZP, Deng AP. A new periodic solution to Jacobi elliptic functions of MKdV equation and BBM equation. Acta Math Appl Sin, English Series. 2012;28(2):409-415.

[53] Acan O, Firat O, Keskin Y, Oturanc G. Conformable variational iteration method. New Trends in Mathematical Sciences. 2017;5(1):172-178.

[54] Acan O, Firat O, Keskin Y. Conformable variational iteration method, conformable fractional reduced differential transform method and conformable homotopy analysis method for non-linear fractional partial differential equations. Waves in Random and Complex Media. 2020;30(2):250-268.

[55] He J. A new approach to nonlinear partial differential equations. Comm. Nonlinear Sci Numer Simulat. 1997;2(4):230235.

[56] He JH. Variational iteration method-a kind of non-linear analytical technique: some examples. Int J Nonlin Mech. 1999;34(4):699-708.

[57] He JH. Approximate analytical solution for seepage flow with fractional derivatives in porous media. Comput Methods in Appl Mech Eng. 1998;167(1-2):57-68.

[58] Wang G, Yang K, Gu H, Guan F, Kara AH. A (2+1)-dimensional sine-Gordon and sinh-Gordon equations with symmetries and kink wave solutions. Nucl Phys B. 2020;953:114956.

[59] Latha MM, Vasanthi CC. An integrable model of (2+1)dimensional Heisenberg ferromagnetic spin chain and soliton excitations. Phys Scr. 2014;89(6):065204. 